\title{
New approaches to SNe Ia progenitors
}

\author{
Pilar Ruiz-Lapuente ${ }^{1,2}$
}

Received _ _

\footnotetext{
${ }^{1}$ Instituto de Física Fundamental, Consejo Superior de Investigaciones Científicas, c/. Serrano 121, E-28006, Madrid, Spain

${ }^{2}$ Institut de Ciències del Cosmos (UB-IEEC), c/. Martí i Franqués 1, E-08028, Barcelona, Spain
} 


\begin{abstract}
Although Type Ia supernovae (SNe Ia) are a major tool in cosmology and play a key role in the chemical evolution of galaxies, the nature of their progenitor systems (apart from the fact that they must content at least one white dwarf, that explodes) remains largely unknown. In the last decade, considerable efforts have been made, both observationally and theoretically, to solve this problem. Observations have, however, revealed a previously ususpected variety of events, ranging from very underluminous outbursts to clearly overluminous ones, and spanning a range well outside the peak luminosity-decline rate of the light curve relationship, used to make calibrated candles of the SNe Ia. On the theoretical side, new explosion scenarios, such as violent mergings of pairs of white dwarfs, have been explored. We review those recent developments, emphasizing the new observational findings, but also trying to tie them to the different scenarios and explosion mechanisms proposed thus far.
\end{abstract}

Subject headings: Supernovae, general; supernovae, Type Ia; thermonuclear explosions; close binaries; white dwarfs 


\section{Introduction}

Type Ia supernovae (SNe Ia) have been the tool that made possible the discovery of the acceleration of the expansion of the Universe (Riess et al. 1998; Perlmutter et al. 1999), and they are now providing new insights on the cosmic component, dubbed "dark energy", thus revealed. However, in contrast with their key role as cosmological probes, and after more than 50 years of supernova research, the nature of their progenitors remains elusive. As far back as 1960, it was established that Type I supernovae (in fact, the now denominated SNe Ia, or thermonuclear supernovae) should result from the ignition of degenerate nuclear fuel in stellar material (Hoyle \& Fowler 1960). The absence of hydrogen in the spectra of the SNe Ia almost immediately suggested that they were due to thermonuclear explosions of white dwarfs (WDs). Isolated white dwarfs were once thought to be possible progenitors (Finzi \& Wolf 1967), but soon discarded due to incompatibility with basic results from stellar evolution. Instead, accretion of matter from a close companion star in a binary system, by a previously formed $\mathrm{C}+\mathrm{O}$ white dwarf with a mass close to the Chandrasekhar mass, provides a viable mechanism to induce the explosion (Wheeler \& Hansen 1971).

Two main competing production channels are still under discussion nowadays. One possible path is the so-called single degenerate $(\mathrm{SD})$ channel, where a $\mathrm{C}+\mathrm{O}$ white dwarf grows in mass by accretion from a non-degenerate stellar companion: a main sequence star, a subgiant, a helium star, a red giant, or an AGB star (Whelan \& Iben 1973; Nomoto 1982). Another possible path is the double degenerate (DD) channel (Webbink 1984; Iben \& Tutukov 1984), where two WDs merge due to the loss of angular momentum by gravitational radiation. The merging could produce the collapse of the white dwarf (Saio \& Nomoto 1985), or it can produce a larger C+O white dwarf configuration that then explodes (Pakmor et al. 2012).

In the decade of the 90's, the variety amongst SNe Ia was discovered, ranging from 
events such as SN 1991bg to those as SN 1991T, through normal SNe Ia (see Filippenko 1997a,b; Branch et al. 2007; Leibundgut 2011). Such diversity was made amenable for cosmology when the correlation of the luminosity at the maximum of the light curve of each SN Ia with its rate of decline was parameterized (Phillips 1993, 1999; Riess, Press \& Kirshner 1995; Perlmutter et al. 1997). It became clear, then, that SNe Ia could be used as distance indicators in cosmology, and that led to the aforementioned discovery.

Yet, the first decade of the new century has brought new surprises: superChandrasekhar supernovae, as well as extremly faint ones (see below). Neither of them are useful for cosmology, although they are not a severe nuisance there, since they can be easily identified, and eliminated from the large samples of SNe Ia collected for cosmological probes. Also, various teams have started to measure supernova rates at a wide variety of redshifts. The idea of using SNe Ia rates to discover the nature of the progenitor systems has now become an active line of research. Finally, high-resolution spectroscopic observations of SN have yielded the surprising result of time-varying absorptions, which indicate the existence of outflows in the circumstellar medium surrounding some $\mathrm{SN}$, and points to possible nova activity previous to the explosion. An intriguing C II feature has been identifieed, close to the Si II line typical of SNe Ia, and that has led to thinking in two different directions: either the thermonuclear flame does not burn the outermost layers of the white dwarf, or maybe $\mathrm{C}$ is a signature of the merged white dwarf companion of the SN. There are also better estimates of the maximum $\mathrm{H}$ mass that could be present in the envelopes of the pre-SNe, if the explosions were triggered by accretion from a non-degenerate companion. There is continued failure to detect $\mathrm{H}$ from the radio emission of the SNe Ia, and there could be constraints from the $\mathrm{X}$-ray emission as well. The task of searching for the companion star in Galactic supernovae has already given some definite results, and there are, now, simulations of the impact of the SN ejecta on the companion star that can be compared with the observations. 
In the following Sections, we present and discuss those new results. In Section 2 we briefly review the different models proposed to explain the SN Ia phenomenon. Section 3 examines how the Delay Time Distribution (DTD) constrains the possible SN Ia progenitors. In Section 4 we discuss the carbon and oxygen absorption features seen, in recent years, in the spectra of SN Ia at early times, while Section 5 deals with the emission features at late times. Section 6 discusses the variable blueshifted sodium feature seen in some SNe Ia. The X-ray constraints are presented in Section 7, and the radio constraints in Section 8. In Section 9 we report the limits on the luminosities of the companions of SNe Ia obtained from pre-explosion images. Section 10 deals with the detection of companions throught the early light curves of SNe Ia. Section 11 reviews the direct searches for surviving companions, in the Galaxy and in the Large Magellanic Cloud. Section 12 deals with the identification of possible candidates to SNe Ia through reconstruction of the orbital evolution of diverse close binary systems containing white dwarfs. Section 13 addresses the important problem of the outliers from the peak brightness-decline rate of the light curve relationship used to make these SNe calibrated candles for cosmology. Section 14 deals with the bulk of SNe Ia used for cosmology. We summarize the current state of affairs in the last Section.

\section{Models}

An ideally complete model of a Type Ia supernova should start from the formation and subsequent evolution of the binary system assumed to originate it, include possible common envelope episodes, mass tranfer stages (generally nonconservative), especially those immediately leading to the explosion, rotational states of the two stars involved, and finally the ignition process and its development into a full thermonuclear explosion (hydrodynamics and nucleosynthesis). From that, light curves and spectra of the emitted

light should be computed, for the different stages of the outburst (extending until the 
nebular phase). The characteristics of the resulting remnants should be predicted as well. In addition, the frequency of the explosions corresponding to the model has to be estimated, for different galactic environments. The whole programme involves very diverse domains of expertise, so the progress has been disperse.

\subsection{Double-degenerate models}

Those models were first proposed by Webbink (1984) and by Iben \& Tutukov (1984). The most favored scenario, in the latter work, started from binaries with component masses in the range 5-9 $M_{\odot}$. They experienced two common envelope stages and ended as a pair of $\mathrm{C}+\mathrm{O}$ white dwarfs, with masses in the range $0.7-1 M_{\odot}$, separated by distances $0.2-0.35$ $R_{\odot}$ and orbiting each other with periods $P$ between $12 \mathrm{~min}$ and $14 \mathrm{hrs}$. The system then losses angular momentum by emission of gravitational waves and the two white dwarfs merge on a time scale ranging from $10^{5}$ to $10^{10} \mathrm{yr}$ (merging of binaries due to the emission of gravitational radiation had already been considered by Tutukov \& Yungelson 1979). The merging would occur through disruption of the less massive component of the system. That component fills its Roche lobe first (larger radius and smaller Roche lobe). The mass transfer, then, should have a runaway character, since the more mass the white dwarf losses, the larger its radius becomes (and the smaller its Roche lobe). The material of the disrupted white dwarf would form a thick disk around the more massive one, which would accrete mass from it until reaching the Chandrasekhar limit and explosively ignite $\mathrm{C}$ at its centre. With the caveat that the effects on the orbit of the two common envelope phases could only be roughly approximated, Iben \& Tutukov (1984) found that the rate of such mergings might, alone, account for the Galactic SN Ia rate.

In the preceding scenario, it was assumed that the only effect of the accretion of matter by the more massive white dwarf, from the debris of its companion, should be growth up 
to the Chandrasekhar mass. That was soon challenged by Saio \& Nomoto (1985), who argued that a very fast mass transfer $\left(\dot{M} \sim 1 \times 10^{-5} M_{\odot} y r^{-1}\right)$ would produce an off-centre C flash. A C-burning front would then propagate (nonexplosively) down to the centre of the white dwarf, changing the chemical composition from $\mathrm{C}+\mathrm{O}$ to $\mathrm{O}+\mathrm{Ne}+\mathrm{Mg}$ along the way. Electron captures on $\mathrm{Mg}$ and Ne would subsequently make the Chandrasekhar mass smaller than the white dwarf mass, and gravitational collapse would ensue. The outcome should thus be the formation of a neutron star, rather than a SN Ia explosion. Nomoto \& Iben (1985) further concluded that the off-centre C ignition would always occur unless the mass accretion rate were less than one-fifth of the Eddington limit for an isolated white dwarf. Later hydrodynamic simulations (Benz et al. 1990; Guerrero et al. 2004) have confirmed that a heavy accretion disk is formed around the most massive white dwarf. Whether a SN Ia ensues would depend on the mass accretion rate of the white dwarf from the disk, that being determined by the viscosity of the latter: a sufficiently low viscosity would allow the white dwarf to grow while avoiding the off-centre, non-explosive ignition of C (Mochkovich, Guerrero \& Segretain 1997). The issue remains open. Another effect of mass acccretion from a massive disk should be the gain of angular momentum by the white dwarf. Its consequences have been studied by Piersanti et al. (2003a,b) and Tornambé \& Piersanti (2013). They find that rotation can stabilize the white dwarf against contraction, even when its mass becomes larger than the Chandrasekhar mass. Later, loss of angular momentum by emission of gravitational waves allows contraction, until the conditions for explosive C ignition are reached at the centre. Recently, Lebanon, Soker \& Garcia-Berro (2014) find constraints on the classical DD type of progenitor from the disk-originated matter around the exploding WD, as this matter will be shocked by the SN ejecta and end up in a radiation implying a larger progenitor radius than observed.

A different approach is based on the violent merging of a pair of white dwarfs (Pakmor et al. 2010). Another possibility is the direct collision of a pair of white dwarfs. Such 
collisions could take place in environments of high star number density, like globular clusters or the Galactic centre (Benz et al. 1989), and also in triple star systems where a close pair of white dwarfs have their orbits perturbed by the third, more distant star (Thompson 2011; Katz \& Dong 2012; Kushnir et al. 2013; Dong et al. 2014). In the hydrodynamic simulations of Pakmor et al. (2010), the merging of two equal mass $\left(\sim 0.9 M_{\odot}\right)$ white dwarfs produced a subluminous SN Ia. Further modeling (Pakmor et al. 2011) has shown that violent mergings where the primary mass is $\sim 0.9 M_{\odot}$ can give rise to subluminous $\mathrm{SNe}$ Ia for unequal masses too, provided that the mass ratio is more than about 0.8. Later on, Pakmor et al. (2012) have found that the violent merging of two $\mathrm{C}+\mathrm{O}$ white dwarfs, with masses $0.9 \mathrm{M}_{\odot}$ and $1.1 \mathrm{M}_{\odot}$ (assumed to induce the detonation of the $\mathrm{C}+\mathrm{O}$ mixture) can account for normal SNe Ia as well. That has been very recently confirmed by García-Senz et al. (2013), who find that the amount of ${ }^{56} \mathrm{Ni}$ produced in the collisions ranges from $0.1 \mathrm{M}_{\odot}$ to $1.1 \mathrm{M}_{\odot}$, thus covering from subluminous through normal up to overluminous SNe Ia. They argue, however, that given the distribution of white dwarf masses, mostly subluminous events should arise. On the other hand, Ruiter et al. (2013) find that the brightness distribution of the explosions produced by violent mergers matches the shape of that observed for SNe Ia (although the issue depends on the occurrence of a particular phase of mass accretion during binary evolution). Very recently, Kromer et al. (2013a) have successfully explained the narrow emission lines of [O I] in the late-time spectra of SN 2010lp (a subluminous SN Ia) by the violent merger of two C+O white dwarfs, with masses of 0.9 and $0.76 \mathrm{M}_{\odot}$. Even more recently, Moll et al. (2013) have found that prompt detonations following the merging of two white dwarfs can not only reproduce both common and overluminous SNe Ia, but also the width-luminosity relation on which the use of these supernovae as cosmological distance indicators is based.

A key point, though, is whether there are or not enough close binary white dwarf binaries to account for the rate of occurrence of SNe Ia. One approach to this problem is to 
look for such systems in the Galaxy. Napiwotzki et al. (2007) have reported the results of a systematic radial velocity survey for double degenerate binaries as potential progenitors of Type Ia supernovae. More than 1000 WDs and pre-white dwarfs were observed with the VLT. The frequency of He WDs is much higher than that of $\mathrm{C}+\mathrm{O}$ WDs, and they regard He WD donors as a possible important channel for SNe Ia. Recently, Badenes \& Maoz (2012), using multi-epoch spectroscopy of $\sim 4000$ white dwarfs from the Sloan Digital Sky Survey, have determined the white dwarf merger rate per unit stellar mass in our Galaxy. They find that the total rate might well account for the SN Ia rate in the Milky Way and galaxies of the same type, but that the rate of merging of pairs of white dwarfs with a total mass above the Chandrasekhar mass is only $\sim 1 / 14$ of the total rate. So, unless sub-Chandrasekhar mergers can produce SNe Ia, the double-degenerate channel should be, at most, a minor contributor to the SN Ia phenomenon.

SN Ia models based on the non-violent merging of two white dwarfs, with a total mass below the Chandrasekhar mass, involve a $\mathrm{C}+\mathrm{O}$ plus a He white dwarf. The He accreted by the more massive $(\mathrm{C}+\mathrm{O})$ white dwarf detonates and the shock wave thus generated can either induce the detonation of the $\mathrm{C}+\mathrm{O}$ layers immediately below or converge near the centre and produce a $\mathrm{C}+\mathrm{O}$ detonation there (Sim et. al. 2012). These authors find that the light curves and spectra of such explosions do match those observed in SNe Ia. Besides, Ruiter et al. (2011) had calculated that if those double-detonation models were able to produce explosions similar to SNe Ia, then the sub-Chandrasekhar explosions would account for a substantial fraction, at least, of the observed SN Ia rate. They also found that the double white dwarf channel involving a $\mathrm{C}+\mathrm{O}$ plus a He white dwarf should have a distribution of delay times (between formation of the binary and the SN explosion) spanning from 800 Myr up to the Hubble time. 


\subsection{Single-degenerate models.}

The channel leading to a SN Ia explosion via mass accretion, by a $\mathrm{C}+\mathrm{O}$ white dwarf, from a non-degenerate binary companion, was first modelled by Whelan \& Iben (1973), although the idea, as we have seen, already appears in Wheeler \& Hansen (1971): a primary with a mass of $1.8-3 M_{\odot}$, plus a secondary with $M \lesssim 0.8 M_{\odot}$, initially form a system with an orbital period between 5 and 9 years. The primary then evolves and becomes a $\mathrm{C}+\mathrm{O}$ white dwarf, with a mass close to the Chandrasekhar mass. The secondary, after $\sim$ $10^{10} \mathrm{yr}$, becomes an AGB star, fills its Roche lobe, and transfers mass to the white dwarf, which then reaches the Chandrasekhar mass and explodes. In this first model, the mass of the secondary was chosen to explain the occurrence of SNe Ia in elliptical galaxies, long

after star formation has stopped. Subsequently, a variety of initial binary systems, in which mass accretion by the white dwarf can take place at different evolutionary stages of its companion (main sequence, subgiant, red giant, AGB), and either from Roche lobe overflow or from a stellar wind, have been proposed. Also, mass loss by the two components of the binary can take place more than once, as well as mass transfer (either conservative or non-conservative). The possibility that the companion might have lost its hydrogen-rich envelope and become a helium star, at the time of mass tranfer to the white dwarf, has also been considered.

A problem common to both the single-degenerate models and to the double-degenerate models, in which the conditions for explosive $\mathrm{C}$ ignition, close to the centre of the $\mathrm{C}+\mathrm{O}$ white dwarf, are reached when the white dwarf mass has grown to the Chandrasekhar mass (or when rotational support has been lost, for masses above such limit), is that thermonuclear burning propagation should be subsonic at first (deflagration) and, at some point, become supersonic (detonation). If only deflagrations were involved, the explosions could just produce a subclass of subluminous SNe Ia (Fink et al. 2014). On the 
other hand, pure detonations of Chandrasekhar-mass white dwarfs would burn the $\mathrm{C}+\mathrm{O}$ mixture to Fe-peak elements entirely, in stark contrast with the observations, that show intermediate-mass elements at and around maximum light. A self-consistent modeling of the deflagration/detonation transition still remains elusive, although steady progress is being made (Woosley 2007; Aspden, Bell \& Woosley 2010; Schmidt et al. 2010; Woosley, Kerstein \& Aspden 2011).

There is another problem that only concerns single-degenerate models of SNe Ia: the possible explosive ignition of the material (hydrogen or helium) accumulated on the surface of the accreting $\mathrm{C}+\mathrm{O}$ white dwarf, before the latter reaches the Chandrasekhar mass. Also, in the case of hydrogen, a high rate of accretion may not lead to explosion but to the formation of an extended envelope, which would be ejected by interaction with the mass-donor star, that terminating the accretion process.

Accumulation of hydrogen on the surface of a white dwarf at a low rate should produce nova-like explosions, in which all the accreted material (and maybe even more) would be expelled (Nomoto 1982; Livio \& Truran 1992). The lower limit on the mass-accretion rate, to avoid explosions and strong flashes, is uncertain and depends on the mass of the white dwarf, but a value $\dot{M} \sim 5 \times 10^{-8} M_{\odot} y r^{-1}$ is often given, although Kercek, Hillebrandt \& Truran (1999), in 3D simulations, obtained steady hydrogen burning for rates as low as $\dot{M} \sim 5 \times 10^{-9} M_{\odot} y r^{-1}$ (see discussion in Hillebrandt \& Niemeyer 2000).

Even if hydrogen is burned steadily or in weak flashes, the resulting helium layer should explosively burn, in a detonation, if the rate of accumulation of helium were $\dot{M} \lesssim 5 \times 10^{-8} M_{\odot} y r^{-1}$ (although this is only true for white dwarf masses $M \lesssim 1.13 M_{\odot}$ ). The same applies to the direct accretion of helium. Lower rates would thus be excluded from the production of Chandrasekhar-mass explosions, but they could lead to sub-Chandrasekhar, edge-lit explosions, instead. 
The explosion of helium shells in accreting white dwarfs as a Type Ia SN mechanism was first proposed by Taam (1980) and Nomoto (1982a) (the "double detonation" model), and numerically studied, in one-dimensional (1D) numerical simulations, by Woosley, Taam \& Weaver (1986), and by Livne (1990). The earliest 2D simulations were made by Livne \& Glasner (1991). Such explosions were further investigated by Woosley \& Weaver (1994), and by Livne \& Arnett (1995). The "robustness" of the double detonation model has been recently checked in 2D and 3D simulations by Moll \& Woosley (2013), whilst the conditions for producing helium detonations have been examined by Woosley \& Kasen (2011). The first evidence of a sub-Chandrasekhar explosion was found by Ruiz-Lapuente et al. (1993) (later confirmed by Mazzali et al. 1997), from modeling the spectra of SN 1991bg. Whether double detonation models produce explosions characteristically similar to those of SNe Ia remains an open question, but Ruiter et al. (2011) find that the helium star channel would have delay times $<500$ Myr ("prompt explosions"), while the double white dwarf channel $(\mathrm{C}+\mathrm{O}$ plus He white dwarf) would have longer delay times, as mentioned above.

Successful accretion of hydrogen, leading to growth of a $\mathrm{C}+\mathrm{O}$ white dwarf up to the Chandrasekhar mass through steady shell hydrogen and helium burning, thus requires high accretion rates. That can be achieved for binary stellar companions at different stages ot their evolution, but if hydrogen accumulates faster than it can be burned, a red giant-like structure should form, soon engulfing the companion and leading to a common-envelope stage. A solution to this problem was found by Hachisu, Kato \& Nomoto (1996): when the mass accretion rate exceeds the maximum rate at which hydrogen can be burned, there is no static envelope solution, and the excess material is blown off in a wind. The optically thick wind solution had been previously found to explain the light curves of nova outbursts by Kato \& Hachisu (1994).

White dwarfs accreting mass at high rates should emit large amounts of radiation in 
the X-ray band, and they should appear as luminous supersoft X-ray sources (van den Heuvel et al. 1992; Di Stefano \& Nelson 1996; Yungelson et al. 1996; Kahabka \& van den Heuvel 1997; Li \& van den Heuvel 1997; Orio 2006). However, as we will see in Section 7 , that might be in conflict with the observed X-ray emission of elliptical galaxies and galaxy bulges.

Another possible channel involving a $\mathrm{C}+\mathrm{O}$ white dwarf plus a non-degenerate companion star is the core-degenerate scenario recently advocated by Soker (2013): a Chandrasekhar or super-Chandrasekhar white dwarf is formed from the merging of a white dwarf with the hot, more massive core of an AGB star. The initial white dwarf is disrupted and its material accreted by the AGB core, that leading to the formation of a rapidly spinning, more massive white dwarf. The delay time till explosion would then be given by

the spin-down time of the new white dwarf. Mergings of a white dwarf with the core of an AGB star, following a common-envelope episode, had also been considered as a production mechanism of SNe Ia by Sparks \& Stecher (1974) and by Livio \& Riess (2003). Soker et al. $(2013,2014)$ have proposed the core-degenerate scenario to explain the characteristics of two very different SNe: PTF11kx and SN $2011 \mathrm{fe}$.

In the preceding, we have not dealt with the especifics of the hydrodynamic modeling of the different types of explosions surveyed, which has reached unprecedented standards of realism (see Hillebrandt et al. 2013). They are being matched by 3D models of the spectra that should arise from the explosions (Baron, Hauschildt \& Chen 2009).

\section{Constraints on progenitors from DTD}

The Delay Time Distribution (DTD) is given by the time evolution of the SN rate that would follow an instantaneous burst of star formation. It is related to the observed rate 
$r_{S N e I a}$ by:

$$
r_{S N e I a}(t)=\int_{0}^{t} R(t-\tau) S F R(\tau) d \tau
$$

where $R(t)$ is the DTD, $S F R$ is the star formation rate, and $t$ and $\tau$ are in the SN rest frame (see, for instance, Ruiz-Lapuente \& Canal 1998).

Early research on SNe Ia rates and DTDs indicated the need of a two-component model at least: one that could be fitted with a short DTD population $\left(\sim 10^{8} \mathrm{yr}\right)$ and another one with a long DTD population (3-4 Gyr) (Scannapieco \& Bildsten 2005; Mannucci et al. 2006; Brandt et al. 2010). Recent studies (Maoz \& Mannucci 2012) suggest that the DTD peaks at the shortest times, as a function $\sim t^{-1}$ (see also Mannucci et al. 2005, and Oemler \& Tinsley 1979). Such a function characterizes the dominance of double degenerate mergings. As already mentioned in the previous Section, Badenes \& Maoz (2012) find that the total merger rate of white dwarf pairs (for sub-Chandrasekhar WDs) is similar to the observed SNe Ia rate (see also Ruiter et al. 2011).

The SNe Ia searches in clusters of galaxies at high redshifts (Barbary et al. 2012) have indicated a $t^{\alpha}$ behavior of the rates, with $\alpha=-1.41_{-0.40}^{+0.47}$, which also favors the WD merging channel. Such best fit value is consistent with measurements of the late DTD in field galaxies (Totani et al. 2008). Most predictions for the SD scenario show a steeper late-time DTD (Greggio 2005; Ruiter et al. 2009; Mennekens et al. 2010), whith $\alpha$ ranging from $\alpha$ $=-1.6$ (Greggio 2005) to $\alpha<-3$ (Mennekens et al. 2010), depending on the details of the scenario and on how the binary evolution is calculated (see, however, Hachisu et al. 2008, and Pritchet et al. 2008). Recently, Bonaparte et al. (2013) have computed the cosmic SN Ia rate, for several cosmic star formation rates and progenitor models, and compared it with the observational data. No firm conclusions can be derived, concerning the SN Ia progenitors, but the existence of prompt SNe Ia, exploding within the first $10^{8} \mathrm{yr}$ after the corresponding systems are formed, is required, althought their fraction should not exceed 
15-20\% of the total, to be consistent with the chemical evolution of the galaxies (see, for an updated view of this subject, the review by Maoz, Mannucci \& Nelemans 2014). Also, from the analysis of the environments of 90 Hubble flow SNe Ia discovered by the Nearby Supernova Factory, Rigault et al. (2013) find evidence of two distinct populations with different ages: one associated with current star formation and another one corresponding to passively evolving environments.

\section{Carbon and oxygen absorption features at early times}

If the SNe Ia explosions would come from white dwarf mergings, one would expect to see carbon and oxygen in the early-time spectra, coming from the surrounding clumps of this material, originated by the merging. With the access to large numbers of SNe Ia, the SN Factory has found C II absorption lines in the spectra of several of them (Thomas et al. 2011). These authors estimate that $22_{-6}^{+10} \%$ of SNe Ia exhibit C II signatures as late as 5 days before maximum light. In some cases one can treat them as spherically symmetric absorptions, in others as "carbon blobs". In the context of explosions from the merging of two $\mathrm{C}+\mathrm{O}$ white dwarfs, the presence of this photospheric carbon at high velocities seems justified. Parrent et al. (2011) have studied both the spherically-symmetric C II absorption and the non-spherically symmetric cases. Altavilla et al. (2007) found evidence of a C blob in the normal SN Ia SN 2004dt. Folatelli et al (2012) have also found evidence of unburnt carbon in SNe Ia from the Carnegie Supernova Project, in 30\% of the objects (see Figure 1).

In the case of the normal SN 2011fe, in the M101 galaxy, the availability of early spectra has allowed to see both C II and O I emissions (Parrent et al. 2012; Nugent et al. 2011). The absorption of O I appears at higher velocities, which suggests that SN 2011fe may have had an appreciable amount of unburned oxygen within the outer layers of the ejecta. Mazzali et al. (2014), from modeling of the spectral evolution, find that 
the high-velocity tail of the ejecta differs from the predictions of both deflagration and delayed-detonation models of the explosion.

Nomoto, Kamiya \& Nakasato (2013) suggest that unburnt C inside the ejecta might just come from asymmetric SNe Ia explosions, in a single-degenerate model, for instance, if off-center ignitions take place (Maeda et al. 2010b). However, it is also possible that unburnt $\mathrm{C}$ would be left inside the ejecta in explosions coming from mergings of $\mathrm{C}+\mathrm{O}$ WDs, even from violent ones. Such carbon would not have been ignited by the detonation that takes place in such mergings (Hicken et al. 2007; Pakmor et al. 2010; Hillebrandt et al. 2013). In that case, we would have a minimum of 30\% of SNe Ia coming from double-degenerate systems.

It is important to study through spectropolarimetry (Wang and Wheeler 2008; Höflich 1991 ; Jeffery 1989, 1990 ) the asymmetry of the C II and O I features. Thus far, line asymmetries have been seen in some SNe Ia, such as SN 2004dt (Wang and Wheeler 2008).

\section{Emission features at late times}

The merging models of $\mathrm{C}+\mathrm{O}$ WDs need testing in the nebular phase. As discussed in Röpke et al (2012), several properties of the merging models could be observable in that phase. In general, the model of (initial) deflagration of a WD when reaching the Chandrasekhar mass leads to a high degree of neutronization, since burning occurs at

densities higher than $2 \times 10^{8} \mathrm{~g} \mathrm{~cm}^{-3}$. Such neutronization can be seen, for instance, from the nebular emission of $\mathrm{Ni}$ II (coming from the stable isotope ${ }^{58} \mathrm{Ni}$ ). In the violent merging of two white dwarfs, instead, the burning occurs at peak densities below $2 \times 10^{8} \mathrm{~g} \mathrm{~cm}^{-3}$ (Röpke et al. 2012), and nebular emission of stable Ni should not be so prominent. In fact, the nebular emission of the decay products of ${ }^{56} \mathrm{Ni}$ is expected to be asymmetric, both in the 


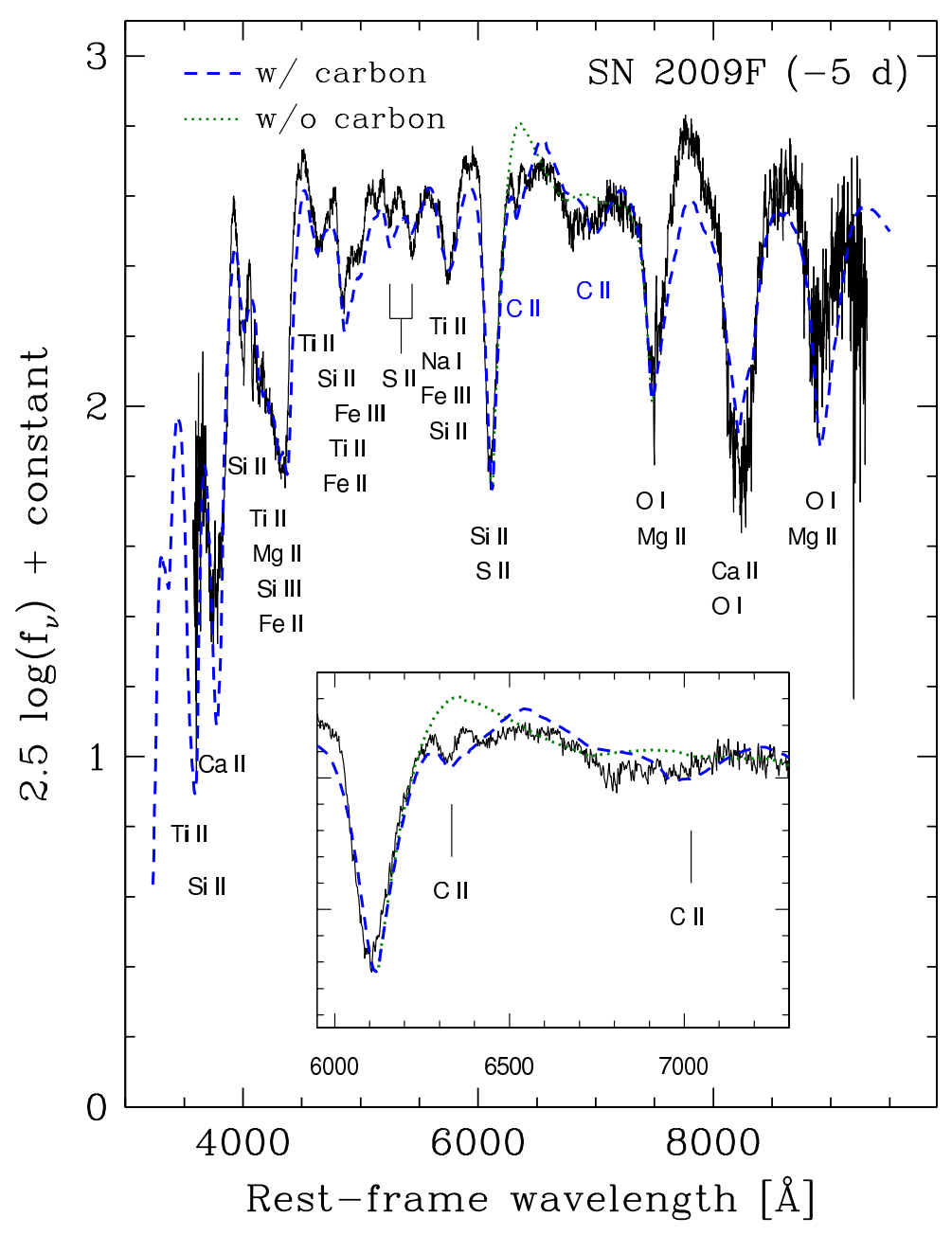

Fig. 1.- Spectrum of SN $2009 \mathrm{~F}$ at -5 days (black solid line) showing the C II $\lambda 6580$ and $\lambda 7234$ lines, and a matching synthetic spectrum from a model with carbon (dashed line). A synthetic spectrum from a model without carbon is shown in dotted lines. (See Folatelli et al. 2012). (Courtesy of Gaston Folatelli. (CAAS. Reproduced with permission). 


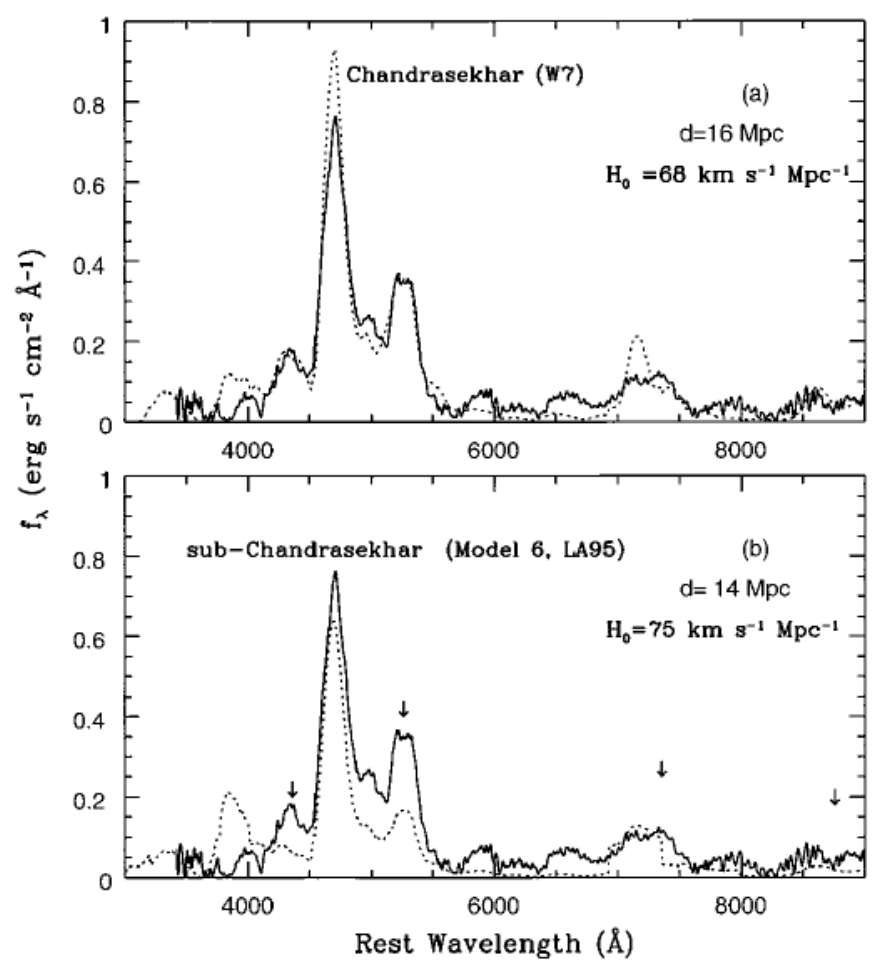

Fig. 2.- Chandraskhar models were favored by normal nebular SNe Ia. Sub-Chandrasekhar He-detonations (Livne \& Arnett 1995) were largely disfavored (Ruiz-Lapuente 1996. C)AAS. Reproduced with permission). 
single degenerate model and in the non-violent merging of two $\mathrm{C}+\mathrm{O}$ WDs, the asymmetry arising from the material produced by a deflagration initiated off-centre, when the WD reaches the Chandrasekhar mass. The Ni produced later, in the detonation phase, would be more symmetrically distributed, instead. Those asymmetries of the nebular emission by the products of ${ }^{56} \mathrm{Ni}$ decay have already been seen, according to Maeda et al. (2010a,b). Maeda et al. (2010a) assert that the nebular spectra do reveal ignitions offset from the centre of the WD, and that this is a generic feature of SNe Ia.

A point made by Röpke et al. (2012) is that, in the violent merging of $\mathrm{C}+\mathrm{O}$ WDs, the detonation of the secondary WD, at low densities, should introduce copious amounts of oxygen in the innermost ejecta. That could give rise to visible [O I] $\lambda \lambda 6300,6364 \AA$. This has been seen in SN 2010lp (Kromer et al. 2013a; Taubenberger et al. 2013a), and it gives strong support to that scenario. A different question (Ruiz-Lapuente 1996) is that, at lower densities, the [Fe II] and [Fe III] lines are less collisionally excited and become weaker than what is seen in the data. This was shown for the sub-Chandrasekhar edge-lit detonations of Livne \& Arnett (1995) which gave a very poor fit to the observations, while Chandraskehar models with $0.6 \mathrm{M} \odot$ of ${ }^{56} \mathrm{Ni}$ fit very well the data of normal SNe Ia. The fit to the nebular data for the new class of sub-Chandrasekhar explosion models remains to be tested, since the result just mentioned came from the models of He detonations in sub-Chandrasekhar WDs available in the 90's (Ruiz-Lapuente 1996, hereafter R96; see Figure 2).

On the other hand, in the models based on Chandrasekhar-mass WDs resulting from accretion of $\mathrm{H}$ from a non-degenerate star, one would expect to see emission of $\mathrm{H}$ at $\lambda 6563$ A. Such emission is not seen in normal SNe Ia (Leonard 2007). Hydrodynamic simulations indicate that seeing the $\mathrm{H} \alpha$ emission is related to the mixing of $\mathrm{H}$ with other elements. In a hydrodynamic simulation for a main-sequence companion to the SN (Liu et al. 2012), up to $19 \%$ of the total mass of the companion star is stripped by the impact of the SN ejecta, 
and those debris should mix with the most slowly moving layers of the ejecta.

There have been a few SNe Ia with $\mathrm{H} \alpha$ in emission. Such are, for instance, the cases

of SN 2002ic (Hamuy et al. 2003), of SN 2005gj (Aldering et al. 2006), and of PTF11kx (Dilday et al. 2012). We will address this point in Section 13.2.

\section{Variable blueshifted Na I D features}

As first seen by Patat et al. (2007), some SNe Ia have variable Na I D absorption lines, significantly blueshifted with respect to the absorption features of other elements. This result has been confirmed by Simon et al. (2009), Sternberg et al. (2011), and Foley et al. (2012) (see Figure 3). The general interpretation is that a significant fraction of SNe Ia progenitor systems have outflows of material previous to the explosion. The Na I D lines arise from the ionized circumstellar medium (CSM). Foley et al. (2012) find a correlation with higher velocity ejecta in the SNe Ia that show blueshifted Na I D line profiles. They suggest the possibility that progenitor systems with strong outflows tend to have more kinetic energy per unit mass than those with weak or no outflows.

Patat et al. (2013) have shown that the SN 2011fe was surrounded by a "clean" environment, and there is a lack of time-variable blueshifted absorption features. They found SN 2011fe consistent with the progenitor being a binary system with a main-sequence or even a degenerate star. Nugent et al. (2011) found that the exploding star was likely a $\mathrm{C}+\mathrm{O}$ WD and, from the lack of an early shock, that the companion was most likely a main-sequence star or there is no surviving companion. Li et al. (2011), from pre-explosion images, also exclude companions more evolved that subgiants (see Section 9). Bloom et al. (2012) also find that only degeneracy-supported compact objects-WDs and neutron stars - are viable as the primary star. With few caveats, they also restrict the companion 
(secondary) star radius to $R_{c} \leq 0.1 R_{\odot}$, that excluding Roche-lobe overflowing red giant and main-sequence companions to high significance.

It would be interesting to see if, within the sample of SNe Ia showing C II and O I absorptions, there are cases of outflows. It is tempting to think that the supernovae with variable Na I D features are connected to nova precursors and the ones showing $\mathrm{C}$ and $\mathrm{O}$ in the outermost layers are connected with mergings of WDs, instead.

Recently, Sternberg et al. (2013) have found that $18 \%$ of the SNe Ia events show time-variable Na I D features associated with circumstellar material. One might tentatively associate them with recurrent novae.

\subsubsection{Recurrent novae as progenitors of SNe Ia}

Recurrent novae are binaries harboring a WD close to the Chandrasekhar mass. Classical novae are the outcome of unstable thermonuclear burning in accreting white dwarfs. Those white dwarfs have typical masses $M \simeq 0.8 M_{\odot}$. If the accretion rate and white dwarf mass are high, the time between flashes can become short enough that the recurrence can be observed. Due to the large accretion rate and insignificant mass loss by

ejection, it has been proposed that in recurrent novae (RNe) the white dwarfs may grow to the Chandrasekhar mass and give rise to SNe Ia (Starrfield et al. 1988; Schaefer 2010).

Indeed, RNe can be the progenitors of a part of the SNe Ia that show variable circumstellar Na absorptions. Amongst recurrent novae, there are those of the U Sco type, where the donor is a main sequence or subgiant star, and those of the RS Oph type, where the donor is a red giant. The supernova PTF11kx, classified as a SN Ia-CSM for its interaction with the circumstellar medium, is believed to have a symbiotic recurrent nova as its progenitor system (Dilday et al. 2012), although it has also been attributed to a 


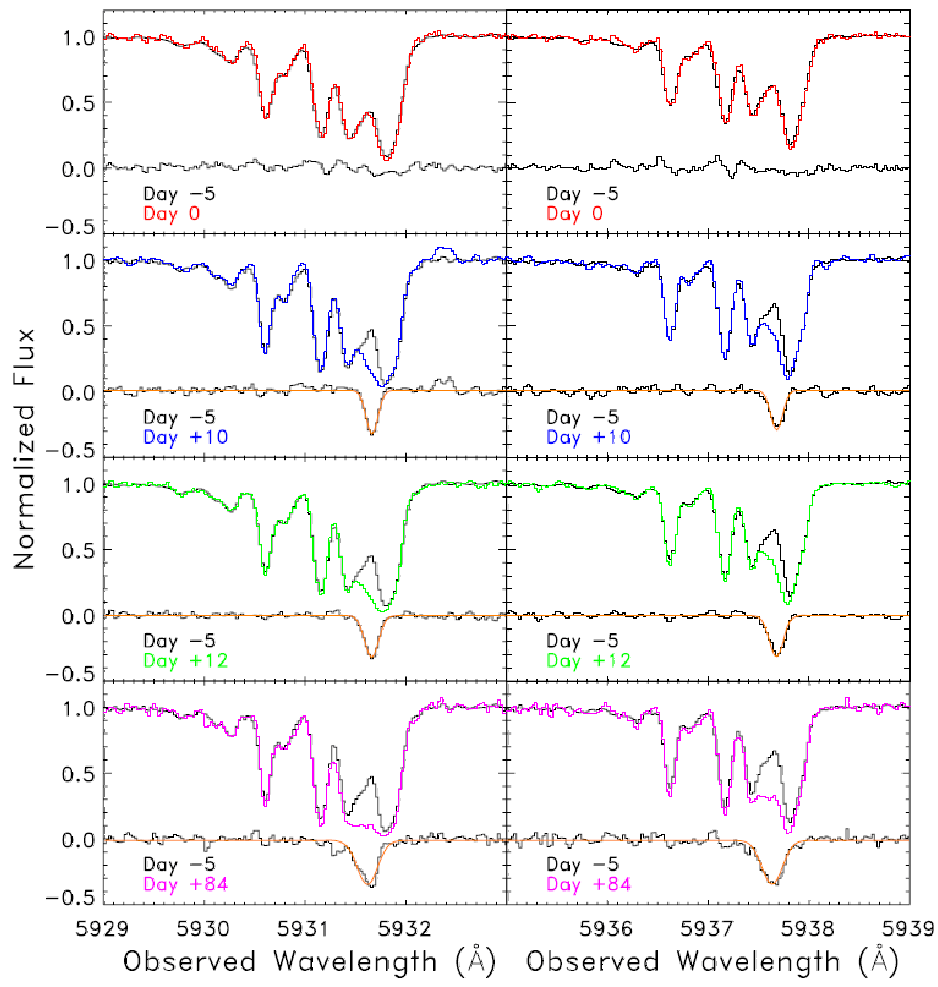

Fig. 3.- High-resolution observations of the Na D absorption lines in the spectrum of SN 2007le (day -5 and subsequent days). Each panel compares with the previous observation and also with the first one (dotted lines). One can see that the $\mathrm{Na}$ absorption line, at $\lambda$ $5931.5 \AA$ A, strengthens with time. (Courtesy of Josh Simon; Simon et al. 2009. C)AAS. Reproduced with permission). 
violent prompt merger of a white dwarf with the core of a massive AGB star by Soker et al. (2013), who also argue that the mass of the shell surrounding PTF11kx is too high to have been produced by a recurrent nova. They estimate the hydrogen mass in the shell to be $M_{s h}>0.2 M_{\odot}$. Also, Lü, Yungelson \& Han (2006) have shown that symbiotic novae are unlikely SNe Ia progenitors, due to their low efficiency in hydrogen accumulation.

Sahman et al. (2013) suggest that the recurrent nova Cl Aql will become a SN Ia within 10 Myr. They find that the mass of the white dwarf is $1.00 \pm 0.14 \mathrm{M}_{\odot}$, and the mass of the companion is $2.32 \pm 0.19 \mathrm{M}_{\odot}$. The radius of the latter is $2.07 \pm 0.06 \mathrm{R}_{\odot}$. They estimate that the secondary is a slightly evolved A-type star, and suggest that the system is rapidly evolving into a supersoft X-ray source. Patat (2011) sees, in the variable sodium lines of some SNe Ia, a possible connection with recurrent novae.

Recently, Soraisam \& Gilfanov (2014) have compared the nova statistics for M31 with the SNe Ia rates. They find that significant mass accumulation, in the unstable burning regime, is only possible for WDs with masses below $1.25 M_{\odot}$. More massive WDs do not significantly accumulate mass. Thus, the final stage of mass growth can not occur at low mass-accretion rates, when the burning is unstable. Therefore, to be SNe Ia progenitor candidates, the systems should go into the stable burning regime in the final phases.

\subsubsection{Other wind-blowing systems made of white dwarfs with main-sequence or subgiant} companions

Systems consisting of a mass-accreting white dwarf and a Roche-lobe filling, more massive, slightly evolved main-sequence or subgiant star, steadily burning H, should appear as luminous supersoft $\mathrm{X}$-ray sources (see Section 2). With varying mass-accretion rates, however, they can also burn $\mathrm{H}$ unstably, at times, and then appear as recurrent 
novae of the U Sco type. Nomoto et al. (2002) (see also Hachisu et al. 1999) propose an scenario in which a $\mathrm{C}+\mathrm{O}$ white dwarf is formed from a red-giant star with a helium core of $\sim 0.8-2.0 M_{\odot}$. Following a first common-envelope episode, a helium star results and then evolves to form a $\mathrm{C}+\mathrm{O}$ white dwarf of $\sim 0.8-1.1 M_{\odot}$. A part of the helium envelope would have been transferred to the main-sequence companion. The white dwarf would thus accrete and burn a mixture of $\mathrm{H}$ and helium. Depending on the mass-accretion rate, a wind might be blown from the surface of the white dwarf. It should not be optically thick enough to absorb all the X-ray emission, but it could, however, absorb a part of the soft X rays (Hachisu, Kato \& Nomoto 2010; see next Section). It will be intersting to see whether variable circumstellar material would be observed in this scenario.

\section{7. $\mathrm{X}$-ray constraints}

The two different channels to SN Ia explosions, the single-degenerate path and the double-degenerate one, lead to very different predictions for the $\mathrm{X}$-ray emission (Gilfanov \& Bogdán 2010). Whereas no strong X-ray emission is expected, prior to explosion, in the merger scenario, in the single-degenerate scenario the white dwarf that accretes mass from a non-degenerate companion becomes a source of X-rays for about $10^{7} \mathrm{yr}$ before the

explosion. If the growth in mass of the white dwarf is due to accretion of hydrogen, followed by steady burning of hydrogen into helium, one expects a thermonuclear luminosity

$$
L_{n u c} \sim \epsilon_{H} X \dot{M} \operatorname{erg~s}{ }^{-1}
$$

where $\epsilon_{H}$ is the energy per unit mass released by hydrogen burning, $X$ is the hydrogen mass fraction in the accreted material, and $\dot{M}$ is the mass-accretion rate. For standard values. $L_{\text {nuc }} \sim 10^{37} \mathrm{erg} \mathrm{s}^{-1}$, which is more than one order of magnitude larger than the gravitational energy released by accretion, $L_{\text {grav }}=G M \dot{M} / R$ ( $M$ and $R$ being the mass and 
radius of the white dwarf). That sustains a surface temperature of the white dwarf:

$$
T_{\mathrm{eff}} \simeq 67\left(\frac{\dot{M}}{5 \times 10^{-7} M_{\odot} / \mathrm{yr}}\right)^{1 / 4}\left(\frac{R_{\mathrm{WD}}}{10^{-2} R_{\odot}}\right)^{-1 / 2} \mathrm{eV}
$$

Such sources are observed in the Milky Way and nearby galaxies, and they are known (see Section 2.2) as supersoft sources (van den Heuvel et al. 1992; Kahabka \& van den Heuvel 1997). Gilfanov \& Bogdán (2010) report that the observed X-ray flux from six nearby elliptical galaxies and galaxy bulges is a factor $\sim 30-50$ less than predicted by the accretion scenario, based upon an estimate of the supernova rate. They conclude that no more than $\sim 5 \%$ of Type Ia supernovae in early-type galaxies can be produced by white dwarfs accreting hydrogen in binary systems. Hachisu, Kato \& Nomoto (2010), however, suggest that there is, in fact, no inconsistency, since symbiotic supersoft sources have fluxes $\sim 0.4 \times 10^{36} \mathrm{erg} \mathrm{s}^{-1}$ in the $0.3-0.7 \mathrm{kev}$ range. There is also uncertainty in theoretically deriving the $\mathrm{X}$-ray luminosity of the supersoft sources, due to the still rough atmosphere models of mass-accreting WDs and to the neglect of absorption of the soft X-rays by the cool wind material from the companion star.

On the other hand, X-ray emission can inform us about the circumstellar medium around the SNe Ia (Badenes et al. 2007). In that work, the authors disfavor optically thick accretion winds from the WD surface. Such winds would produce large cavities in the interstellar medium (ISM). The fundamental properties of the seven supernova remnants (SNRs) of type Ia of their sample (SN 1885, Kepler, Tycho, SN 1006, 0509-67.5, 0519-69.0, and N103B) are incompatible with SNR models expanding inside such cavities. In general, the search for $\mathrm{X}$-ray emission at the time of the supernova outburst has also provided probes of the circumstellar medium, which so far is considered to be of low density (Hughes et al. 2007). Recently, Rusell \& Immler (2012) have examined 53 SNe Ia observed with the Swift X-ray telescope, and their upper limit to the X-ray emission gives further evidence that the companion stars in SNe Ia are neither massive nor evolved (post main-sequence), 
due to the corresponding limit on wind mass-loss rate inferred. They can not rule out, instead, main-sequence star companions, with mass-loss rates $<10^{-7} M_{\odot} y r^{-1}$. A double white dwarf system is also permitted, due to the lack of circumstellar interaction and hence lack of X-rays there.

The tightest constraint on the progenitor of a SN Ia, coming from X-ray emission, is that for SN 2011fe (Horesh et al. 2012; Margutti et al. 2012). The X-ray observations yield an upper limit $2 \times 10^{-9} \mathrm{M}_{\odot} \mathrm{yr}^{-1}$ to the mass outflow (assuming a wind velocity $v_{\text {wind }}=$ $100 \mathrm{~km} \mathrm{~s}^{-1}$ ). As we have seen in Section 2, accretion at a rate $\sim 10^{-7} \mathrm{M}_{\odot} \mathrm{yr}^{-1}$ is thought to be necessary for stable accretion and nuclear burning on the surface of a white dwarf (Nomoto 1982). Supersoft sources can achieve those rates (Kahabka \& van den Heuvel 1997), although the rates can also be either lower or higher, in these sources. Horesh et al. (2012) analyse the models of interaction of the wind with the circumstellar material and conclude that the data from SN 2011fe can rule out a symbiotic system, but not a main sequence or subgiant mass-donor. The same conclusion is reached from their analysis of the radio emission (see next Section). Margutti et al. (2012) also discard symbiotic systems, as well as Roche lobe overflowing subgiants and main-sequence secondary stars if $\geq 1 \%$ of the transferred mass is lost at the Lagrangian points.

The nearby SN 2014J has provided a new opportunity to test the presence of X-ray emission from the pre-explosion X-ray images (Nielsen et al. 2014). According to these authors, the upper limits from the Chandra X-ray observatory do exclude a classical super-soft source as the progenitor. Near the Chandrasekhar mass, the effective temperature corresponding to the stable nuclear burning on the WD surface exceeds $100 \mathrm{eV}$. For this temperature, the $3 \sigma$ upper limit on the bolometric luminosity is $\simeq 3.8 \times 10^{37} \mathrm{erg} \mathrm{s}^{-1}$, assuming a column density of hydrogen $N_{H}=6.9 \times 10^{21} \mathrm{~cm}^{-2}$ and a black body spectrum. That confidently excludes a classical super-soft source during the final stages of the mass 
accumulation by the progenitor. Due to the large absorption, the Chandra upper limits are less constraining at lower temperatures. They do not exclude, therefore, less conventional progenitors, e.g. a WD enshrouded in an optically thick envelope or wind. Deep X-ray observations of the post-explosion environment (Margutti et al. 2014) now rule out single-degenerate progenitors with steady mass loss until the time of the explosion (the maximum mass spilled by the system should be $\leq 1 \%$ ), and do only allow recurrent novae with a recurrence time $<300$ yrs, stars where the mass loss ceases before the explosion, or double WD systems.

From a different angle, there have been, from the start, great expectations to detect hard X-ray and $\gamma$-ray photons from SN 2014J (Isern et al. 2013; The \& Burrows 2014), since the supernova was close enough to be detected by INTEGRAL and NuSTAR. It has, indeed, been detected by INTEGRAL (Churazov et al. 2014). The line flux suggests that $0.62 \pm 0.13 M_{\odot}$ of radioactive ${ }^{56} \mathrm{Ni}$ have been synthesized in the core. The mass of the ejecta (from the continuum emission) would be $\sim 1.4 M_{\odot}$ and composed of roughly equal fractions of iron-group and intermediate-mass elements. There is thus agreement with the model of the explosion of a Chandrasekhar-mass WD. Diehl et al. (2014) find that

about $0.06 \mathrm{M}_{\odot}$ of ${ }^{56} \mathrm{Ni}$ should be at the outskirts of the ejecta. This has suggested that He accreted by the white dwarf could have exploded in the external layers and triggered the central ignition.

\section{Radio emission}

The lack of radio emission from Type Ia supernovae has been useful in discarding one type of single-degenerate path as a major contributor: SNe Ia from symbiotic systems. In symbiotic systems, the white dwarf accretes mass from the wind of a giant or AGB companion. The wind accretion should produce radio emission when the SN ejecta interact 
with the circumstellar environment created by such systems. Panagia et al. (2006) set upper limits on mass-loss rates of $\sim 10^{-7} \mathrm{M}_{\odot} \mathrm{yr}^{-1}$. Hancock et al (2011) suggest upper limits, to the average mass-loss rate of the companion by stellar wind, of $1.3 \times 10^{-7} M_{\odot} y r^{-1}$. These authors say that such limit is inconsistent with SNe Ia in which the accretion comes from intermediate or high-mass companions. Instead, a main sequence star having fast winds $\left(>10 \mathrm{~km}^{-1}\right)$ could remain undetected, even with much higher mass-loss rates.

The nearby supernova SN 2011fe has made possible the most sensitive radio study of a SN Ia made up to now (Chomiuk et al. 2012). The data set direct constraints to the density of the surrounding medium at radii $\sim 10^{15}-10^{16} \mathrm{~cm}$, that implying an upper limit on the mass-loss rate from the progenitor system of $\dot{M} \lesssim 6 \times 10^{-10} M_{\odot} y r^{-1}$ (assuming a wind speed of $100 \mathrm{~km} \mathrm{~s}^{-1}$ ), or expansion inside a uniform CSM with density $\lesssim 6 \mathrm{~cm}^{-3}$. Drawing from the observed properties of non-conservative mass transfer in accreting white dwarfs, they use the limits on the density of the circumstellar environment to exclude a good fraction of the parameter space of possible progenitor systems of SN 2011fe. A symbiotic progenitor system can be ruled out, as well as any other system characterized by a high mass-transfer rate onto the white dwarf which could give rise to optically thick accretion winds. Assuming that a small fraction, $\sim 1 \%$ of the mass transferred, is lost from the progenitor system, they can also eliminate much of the parameter space occupied by potential progenitors such as recurrent novae or, alternatively, progenitors undergoing stable nuclear burning. They eliminate, therefore, for SN 2011fe, a large fraction of the parameter space associated with popular single-degenerate progenitor models, leaving only a limited region, mostly inhabited by some double degenerate systems, as well as by exotic single degenerates in which a sufficient time delay takes place between mass accretion and SN explosion.

The even closer SN 2014J has also been observed in radio with the VLA, without any 
detection (Chandler \& Marvil 2014). This points out to a surrounding medium of low density as well.

\section{Limits from pre-explosion images}

It has been possible to put constraints on the progenitors from pre-explosion images in other galaxies. This endeavour has been of particular interest for SN 2011fe, since it exploded in the galaxy M101, at 6.4 Mpc only. Another nine SNe Ia with preexisting HST data on their host galaxies have also been close enough (within $25 \mathrm{Mpc}$ ) to search for the progenitors. It has only been possible to set upper limits which rule out normal stars with initial masses larger than $6 \mathrm{M}_{\odot}$ at the tip of the AGB branch, young post-AGB stars with initial masses larger than $4 \mathrm{M}_{\odot}$, and post-red-giant stars with initial masses above $9 \mathrm{M}_{\odot}$ (Li et al. 2011a).

The case of SN 2011fe arose great expectations, since the SN was much closer than in previous occasions. There was, however, no object seen at the location of the supernova in pre-explosion images, down to magnitude 27.4 (in the ACS/F435W band) (Li et al. 2011a). The conclusion of the analysis is that, for SN 2011fe, the red giant progenitor is excluded, while a subgiant or a main-sequence companion star still are possible progenitors, from the imaging approach.

Very recently, the nearby supernova SN 2014J in M82 has been tested in the same way. This supernova is only at 3.5 Mpc. Elias-Rosa, Greggio \& Botticella (2014) have analyzed deep archival HST WFC3/IR images of M82 in the F110W and F160W filters, taken in Jan. 2010, and used them in an attempt to identify a progenitor for the SN, by registering the HST images with images of the SN taken on Jan. 23 2014. As in the SN 2011fe case, they can again exclude a red-giant companion. The limits are consistent with the companion 
being (if not another WD) a subgiant or a main-sequence star. Goobar et al (2014) have also explored the HST images of the explosion region. The observational limits, however are not as constraining here as in the case of SN 2011fe.

\section{Seeing the companion through the early light curve}

According to Kasen (2010), the impact of the supernova debris on the companion produces a bright $\mathrm{X}$-ray $(0.1-2 \mathrm{keV})$ burst lasting from minutes to hours. The diffusion of this X-ray emission gives rise to a longer-lasting optical/UV emisson which exceeds the radioactively powered emission from the supernova for the first few days after the explosion. This effect can be seen in Figure 4. The signatures are prominent for viewing angles looking down upon the shocked region, which should be about a $10 \%$ of the times. Kasen (2010) concludes that the current optical and UV data do effectively constrain the red giant companion channel, disfavoring it.

Zheng et al. (2014) (see their Figure 3), have presented very early light-curve data from SN 2014J. When comparing them with the predictions of Kasen (2010), the non-companion case appears favored.

High-z searches might provide the tightest constraint on the SN progenitors. Goldhaber et al. (2001) show, in their Figure 1, the light curves of 35 high-z SNe Ia found by the Supernova Cosmology Project (SCP). These data, as well as more recent ones (Conley et al. 2006; Hayden et al. 2010; Bianco et al. 2012) do not show any evidence for a companion in the early light curves of high-z SNe Ia samples. Hayden et al. (2010), from a simulation of the shock interaction with a companion, rule stars with masses larger that $6 \mathrm{M}_{\odot}$ and also disfavour red-giant companions. 

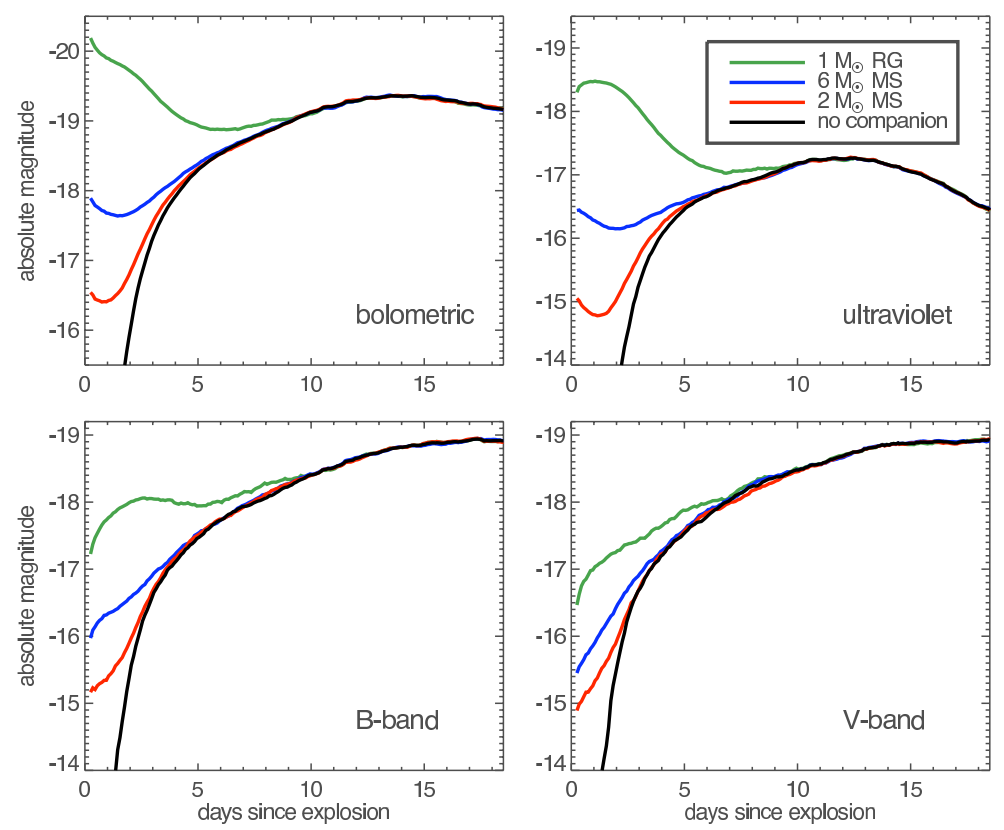

Fig. 4.- Differences in the early light curve of a SN Ia, depending on the nature of the companion star, from Kasen (2010). Four possible progenitor scenarios are modelled: a RG companion at a distance $a=2 \times 10^{13} \mathrm{~cm}$ (green lines); a $6 \mathrm{M}_{\odot} \mathrm{MS}$ companion at $a=2 \times 10^{12} \mathrm{~cm}$ (blue lines); a $2 \mathrm{M}_{\odot}$ MS companion at $a=5 \times 10^{11} \mathrm{~cm}$ (red lines), and the lack of companion (black lines). (Courtesy of Dan Kasen. CAAS. Reproduced with permission). 


\section{Search for companion stars of SNe Ia in our Galaxy and in the LMC}

Another method to identify the progenitors of the SNe Ia was proposed by RuizLapuente (1997): to inspect the stars within the innermost regions of the Galactic SNe Ia remnants in search of the mass-donor star (to either find it or to show its absence), in the area where it should still remain after the explosion, moving with a peculiar velocity gained from the orbital velocity in the binary system before the explosion. A given star, to be candidate to donor in a SN Ia explosion, should be at the distance of the remnant, moving with enhanced velocity, and maybe also show signs of contamination by the iron-peak rich part of the supernova ejecta. A subgiant named Tycho $\mathrm{G}$ was found to be a likely candidate companion for SN 1572 (Ruiz-Lapuente et al. 2004), since it is close to centre of the SNR, at a distance compatible with that of the remnant, and it is in a region where stars follow the rotational pattern of the Galaxy, but it has a radial velocity well above the 20 to 40 $\mathrm{km} \mathrm{s}^{-1}$, typical at the distance of SN 1572. It also has a high proper motion. A chemical analysis of the star showed enhancement of Ni in the surface (González Hernández et al. 2009), suggesting contamination by the supernova ejecta. That was disputed by Kerzendorf et al. (2009, 2013), who argued that all those characteristics might just correspond to a chance interloper. Based on greatly improved proper motion measurements and a more refined chemical analysis, Bedin et al. (2014), however, have shown that the probability of having found such an interloper at random is extremely low.

Schaefer \& Pagnotta (2012) have looked as well for a companion star in a SNR of the LMC (SNR 0509-67.5) and found no star that could have been the mass-donor in the progenitor system. Their result points to the supernova having resulted from merging of two white dwarfs. In addition, Edwards, Pagnotta \& Schaefer (2012) have examined the innermost area of the remnant SNR 0519-69.0, also in the LMC, and eliminated red giants, subgiants, and He stars as possible companions of the SN. 
González Hernández et al. (2012) (see also Kerzendorf et al. 2012) have inspected the remnant of the Galactic SN 1006, determining distances and chemical abundances for all candidate stars within the innermost $27 \%$ of the area of the remnant. The lack of detection of any viable candidate star rules out red giant and subgiant stars, as well as any star brighter than $\mathrm{M}_{V} \sim+4.9$ (approximately equal, or slightly less than the solar luminosity).

The key point, from the theoretical point of view, is that all groups that have simulated the impact of the ejecta of a supernova on its companion star (Marietta, Burrows \& Fryxell 2000; Pakmor et al. 2008; Pan, Ricker \& Taam 2012a; Liu et al. 2012, 2013) consistently find that the companion survives the explosion. This important conclusion is the basis for the observational searches.

There has been debate on whether the surviving companion of a WD plus main sequence system, or a WD plus subgiant system, would show rapid rotation after the explosion (González Hernández et al. 2009; Kerzendorf et al. 2009, 2013; Bedin et al. 2014). Hydrodynamic simulations by Pan, Ricker \& Taam (2012a), for main sequence companions, show that they would lose about half of their initial angular momentum, their rotational velocity dropping to a quarter of the original rotational velocity. The simulations by Liu et al. (2012), also for a companion on the main sequence, equally show that its rotational velocity can be significantly reduced by the effects of the impact of the SN ejecta, falling to a $32 \%-14 \%$ of its pre-explosion value, due to remotion of $55 \%-89 \%$ of the initial angular momentum, taken away by the material stripped during the interaction with the supernova ejecta. It is easy to see (Fig. 1 of Marietta, Burrows \& Fryxell 2000, for instance) that, in the case of a $1.1 \mathrm{M}_{\odot}$ subgiant, remotion of $\sim 0.15 M_{\odot}$ by the impact of the ejecta means reducing the radius of the star, immediately after the impact, to about $1 / 3$ of its previous value only (in front of about $1 / 2$ in the main sequence case), so the drop in rotational veocity must be correspondly larger. 
In their simulations, Pan, Ricker \& Taam (2012a) find that the contamination with $\mathrm{Ni}$ in the companion star, from the passage of the SN ejecta, is of $\sim 10^{-5} M_{\odot}$, for a main sequence star, and of $\sim 10^{-8} M_{\odot}$ for a red giant.

Another point concerns the luminosity to be expected, for the surviving companions of recent SNe Ia. Podsiadlowski (2003) found that, in the case of a subgiant, the star, $10^{3}-10^{4}$ yr after the explosion, might be either significantly overluminous or underluminous, that depending on the amount of heating and the amount of mass stripped, as well as on the previous binary mass transfer. More recently, Shappee, Kochanek \& Stanek (2013) have claimed that, in the case of a main sequence companion (and maximizing the heating), the object should remain significantly overluminous for the above time lapse, but the more realistic simulations of Pan, Ricker \& Taam (2012b), also for a main-sequence star, predict luminosities much closer to that of Tycho $\mathrm{G}, \sim 500 \mathrm{yr}$ after the explosion. In the case of a subgiant, a larger fraction of the material should be directly stripped by the shock wave generated by the impact of the SN ejecta, and there should be less heating of the fraction of the envelope that remains bound.

In all the preceding considerations, it has been implicitly assumed that there is no significant delay between the accretion phase that brings the white dwarf to the Chandrasekhar mass and the SN explosion. That has been questioned by Di Stefano, Voss \& Claeys (2011), who propose a model in which the $\mathrm{C}+\mathrm{O}$ white dwarf, spun-up by accretion of matter and angular momentum, is able to sustain a mass above the Chandrasekhar mass, and only reaches the conditions for explosive C burning when it has lost enough angular momentum, on a time scale that may be long enough to allow the companion star to evolve to the white dwarf stage. Also, overcoming the Chandrasekhar-mass limit would allow exhaustion of the envelope of the companion star, only its compact core remaining at the end of the mass-transfer phase. Based on that, Di Stefano \& Kilic (2012) argue that the 
lack of evidence of ex-companion star in the above mentioned SNR 0509-67.5 does not

mean that such companion does not exist, since it could have become a $\mathrm{C}+\mathrm{O}$ or a He white dwarf by the time of the explosion. The problem of the time scale of spin down of the primary white dwarf has been very recently addressed by Meng \& Podsiadlowski (2013), who obtain an upper limit of a few $10^{7} \mathrm{yr}$. Such times would still allow a companion star to become dimmer than the upper limit set by Schaefer \& Pagnotta (2012), according to Di Stefano and Kilic (2012).

Another remnant being now studied is the Kepler SNR (SN 1604). From the lack of bright stars in the field, Kerzendorf et al. (2014) have ruled out red giants as possible companions of SN 1604. VLT observations with FLAMES of the stars in more than $20 \%$ of the inner core of the SNR have now been granted (Ruiz-Lapuente et al. 2014). It will be very interesting to see what high-resolution spectra reveal. Other fairly symmetrical Galactic SNR are planned to undergo a similar scrutiny

\section{SN Ia candidates from orbit reconstruction}

Another approach to dilucidate the progenitors of SNe Ia is to reconstruct the orbit of systems containing a WD. Along these lines, the tight binary system CD-30 11223 has been found to consist of a $\mathrm{C}+\mathrm{O}$ white dwarf plus a hot helium star (Geier et al. 2013). The system turns out to be a progenitor candidate for the double detonation SN Ia scenario (see Section 2.2). Wang \& Han (2012) have studied this kind of possible progenitor system (see also Wang et al. 2009). A C+O white dwarf is first formed, from the initially more massive star, and the system then is in a close orbit. The mass donor later reaches Roche-lobe overflow and becomes a He star, but evolves, after exhaustion of the central He, to the red giant stage. The system thus becomes a $\mathrm{C}+\mathrm{O}$ WD plus a He red giant. The mechanism can also work with the He star still staying in the main sequence phase. Fink, Hillebrandt 
\& Röpke (2007) reproduce, in a 3D simulation of a double detonation, these explosions. They find that the He detonation in a shell succesfully gives rise to a second detonation in the $\mathrm{C}+\mathrm{O}$ core. In the outcome, ${ }^{56} \mathrm{Ni}$ masses about $0.40-0.45 \mathrm{M}_{\odot}$ are produced, with rapidly expanding ${ }^{56} \mathrm{Ni}$ in the outer layers. They note, however, the lack of observations of this type of explosion (SN 1991T could resemble it, but the core contained $0.8 \mathrm{M}_{\odot}$ of ${ }^{56} \mathrm{Ni}$ ).

A $\mathrm{C}+\mathrm{O}$ WD plus a He donor could, instead, be the progenitor of the so-called "Type .Ia" supernovae. We do not include these in our physical diagram for SNe Ia, however, since their likely He features at maximum (Kasiwal et al. 2010, and references therein) rule them out as possible SNe Ia (we only consider, in this paper, the types of explosions, either total or partial, that by their features at maximum can be regarded to be such).

\section{Outliers from the brightness-decline rate relation and the bulk of SNe Ia}

From the many systematic searches made at various redshifts, it has been possible to identify SNe Ia that fall well away from the Phillips $(1993,1999)$ relationship between the peak luminosiy and the rate of decline of the light curve. Such relationship was traditionally related to the amount of ${ }^{56} \mathrm{Ni}$ synthesized in Chandrasekhar-mass models, since its variation not only correlated with that of the maximum luminosity, but it also produced opacity variation in the envelope of the $\mathrm{SN}$, which resulted in slower decline rates of the light curve for larger Ni masses. In such view (Höflich \& Khokhlov 1996; Pinto \& Eastman 2000; Bravo

et al. 2009), only variations among Chandrasekhar-mass WD explosions were the cause of the relationship. A new proposal, which completely changes the explanation, is to assume that the Phillips relationship results from variation in the viewing angle of the family of detonations of merging sub-Chandrasekhar explosions (Moll et al. 2013). That marks a new turn in the search for the physical basis of a relationship that is crucial for cosmology, and it thus calls for further investigation. Since this is relevant for cosmology, we mention 
that the method of determination of $\mathrm{H}_{0}$ using nebular spectra of SNe Ia (Ruiz-Lapuente 1996) favored a value of $68 \mathrm{~km} \pm 7$ (stat) \pm 1 (updated systematic error) $\mathrm{km} \mathrm{s}^{-1} \mathrm{Mpc}^{-1}$, in good agreement with the latest results from the Planck satellite (Ade et al. 2013). The light curves of SNe Ia (Höflich \& Khokhlov 1996) favored $67 \pm 9 \mathrm{~km} \mathrm{~s}^{-1} \mathrm{Mpc}^{-1}$ (also in agreement with Planck). Riess et al. (2011), within the SHOES program, had found $H_{0}=73.8 \pm 2.4 \mathrm{~km} \mathrm{~s}^{-1} \mathrm{Mpc}^{-1}$, in tension with the Planck result (but see, more recently, Riees 2014).

\subsection{Super-Chandrasekhar SNe Ia}

A few discoveries of highly luminous $\left(M_{V} \sim-20.4\right)$ SNe Ia suggest the existence of super-Chandrasekhar mass explosions (Howell et al. 2006). These supernovae show very slowly evolving Si II $\lambda 6355 \AA$ absorption velocity, and they can also show a plateau in their blue light curve (Scalzo et. al. 2012). The very large mass of ${ }^{56} \mathrm{Ni}$ needed to explain those events can plausibly be produced by the collision of two white dwarfs (Raskin et al. 2010) or by accretion on a rapidly rotating $\mathrm{C}+\mathrm{O}$ WD. The $S N$ Factory has tried to evaluate the percentage of super-Chandrasekhar SNe Ia, and they suggest about 2\% (Aldering 2011). A super-Chandraskhar WD can be formed if it is supported by rapid rotation (Hachisu 1986; see also Section 10), and the rotating WD is more massive than a non-rotating WD with the same central density (Yoon \& Langer 2005). For a given central density, the density profile is shallower for those more massive WDs, and therefore the mass contained within the density range for ${ }^{56} \mathrm{Ni}$ production is larger. Also, for the same central density, a flame

produces more ${ }^{56} \mathrm{Ni}$ due to less pre-expansion ahead of the propagating flame. In the case of merging, one can have tamped detonations (Howell et al. 2006). Those tamped detonations in rapidly rotating WDs can synthesize amounts of ${ }^{56} \mathrm{Ni}$ as high as $1.6-2 \mathrm{M}_{\odot}$. Taubenberger et al. (2011) have estimated that the total mass of the WD, in the case of SN 2009dc, was 
$\sim 2.8 M_{\odot}$, and the ejected ${ }^{56} \mathrm{Ni}$ mass was $\sim 1.8 M_{\odot}$.

The fact that some of those events show C II absorption features in their spectra reinforces the hypothesis that they come from mergings of two WDs.

On the other hand, from modeling (Hillebrandt et al. 2013) of the violent WD merger scenario (Pakmor et al. 2010), it seems unlikely that these supernovae would come from violent double-degenerate mergers. The ${ }^{56} \mathrm{Ni}$ mass produced only depends on the mass of the primary WD, there. Since exploding $\mathrm{C}+\mathrm{O}$ WDs, in the violent merger model, usually have masses well below $1.3 \mathrm{M}_{\odot}$ (Ruiter et al. 2013), that limits the amount of ${ }^{56} \mathrm{Ni}$ produced in the explosion to $\sim 1 M_{\odot}$ only. However, recent analyses of the nebular spectra of super-Chandrasekhar events (Taubenberger et al. 2013a), the case of SN 2009dc in particular, indicate that those outbursts can be explained by a merger of two massive $\mathrm{C}+\mathrm{O}$ white dwarfs, producing $\sim 1 \mathrm{M}_{\odot}$ of ${ }^{56} \mathrm{Ni}$ and $\sim 2 \mathrm{M}_{\odot}$ of ejecta. That would come from the explosion of a Chandrasekhar-mass white dwarf, enshrouded by $0.6-0.7 \mathrm{M}_{\odot}$ of $\mathrm{C}+\mathrm{O}$-rich material.

\subsection{Supernovae strongly interacting with the CSM}

There is a fraction of supernovae which show narrow hydrogen emission lines. They were first noticed by Hamuy et al. (2003), in SN 2002ic (see Figure 3). Such SN have been labelled in various ways, until recently being dubbed SNe Ia-CMS (Silverman et al. 2013). The existence of the SN Ia-CSM class of objects seems to indicate that at least some $\mathrm{SNe}$ Ia do arise from the SD channel, since a H-rich CSM can form during the evolution of SD systems. Hamuy et al. (2003) suggest that SN 2002ic could have arised from a binary system containing a $\mathrm{C}+\mathrm{O}$ white dwarf plus a massive $\left(3-7 M_{\odot}\right)$ AGB star, where the total mass loss in $\mathrm{H}$ can reach a few solar masses, since their analysis of the narrow component 


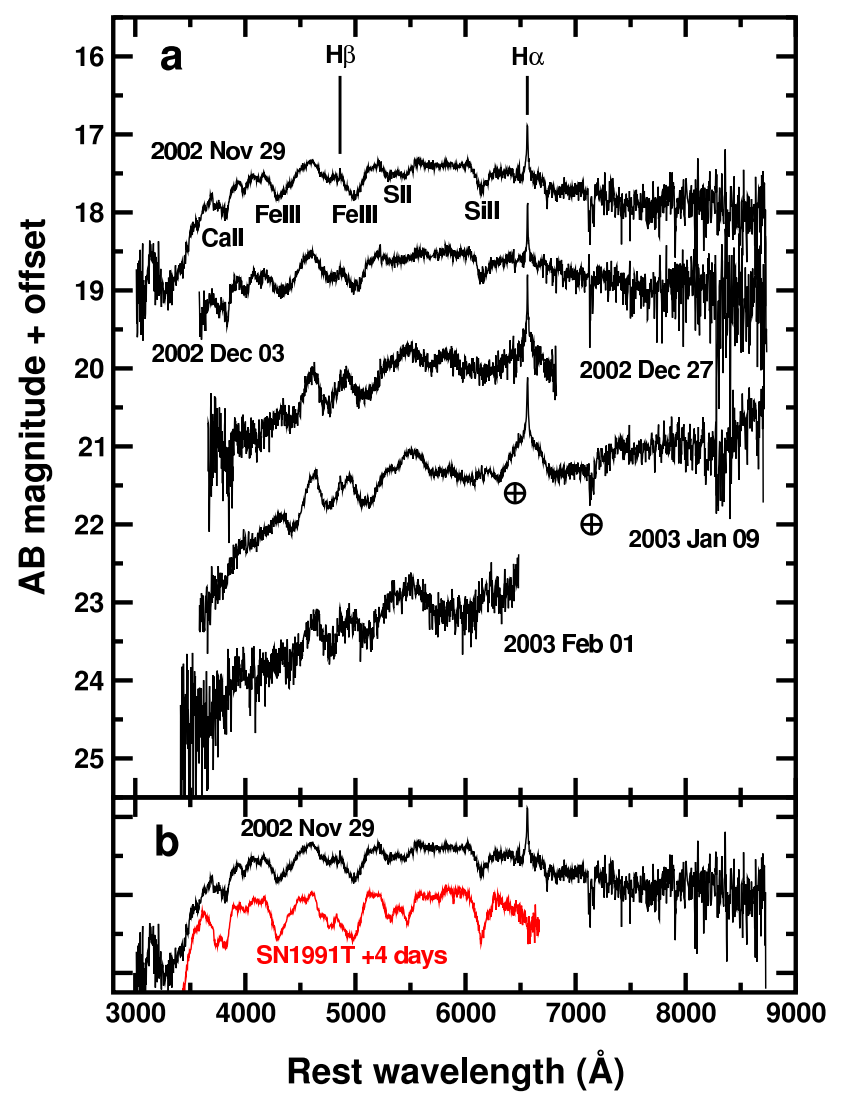

Fig. 5.- Spectroscopic evolution of SN 2002ic (see Hamuy et al. 2003). The spectra were taken at Las Campanas Observatory. In panel a, the evolution for $+6,+10,+34,+47$, and +70 days from estimated maximum light is shown. The top spectrum displays the $\operatorname{Si}$ II $\lambda$ $6355 \AA$ feature typical of the SNe Ia class, as well as prominent Fe III absorption features at $\lambda \lambda 4200$ and $4900 \AA$. In panel b, a comparison of the spectrum of SN 2002ic at the epoch +6 days from maximum with a spectrum of SN 1991T, obtained at the +4 days epoch, shows similarity, except for the $\mathrm{H} \alpha$ emission, which is not present in SN 1991T. (Courtesy of Mario Hamuy. (C)Nature Publishing Group. Reproduced with permission). 
of $\mathrm{H} \alpha$ implies a high mass-loss rate of $\sim 10^{-2.4} M_{\odot} y r^{-1}$ (see Figure 5). The accreted mass would come from the wind of the AGB star, partially captured by the WD. Despite the mass-loss rate being so high, however, the supernova has not been detected in radio.

Dilday et al (2012) find that the supernova PTF11kx is of Type Ia, and suggest a symbiotic nova progenitor (see, however, Soker et al. 2013). Its late-time spectrum confirms that it is, indeed, a SN Ia. A time series of high-resolution spectra of this supernova reveals a complex circumstellar environment, with multiple shells similar to those ejected by Nova RS Ophiuchi. Dilday et al. (2012) found, from the Palomar Transient Factory (PTF), that the SN Ia-CSM are about $0.1-1 \%$ of all SNe Ia. This is more or less consistent with the theoretical expectations for the fraction of SNe Ia from the symbiotic progenitor channel: between 1 and 30\% (Han et al. 2004; Lü et al. 2009).

Concerning other typical characteristics, SNe Ia-CSM have peak absolute magnitudes in the range $-21.3 \leq M_{R} \leq-19 \mathrm{mag}$, with relatively long rise times of $\sim 20-40$ days. They do not emit neither in radio nor at $\mathrm{X}$-ray wavelengths.

\subsection{Types of subluminous SNe Ia}

\subsubsection{SNe Ia of the SN 1991bg-type}

SN 1991bg came as a suprise, being a subluminous SN Ia, one order of magnitude fainter than normal SNe Ia (Filippenko et al. 1992; Leibundgut et al. 1993; Ruiz-Lapuente et al. 1993). The amount of ${ }^{56} \mathrm{Ni}$ synthesized was only about $0.07 M_{\odot}$ (Ruiz-Lapuente et al. 1993). It is clearly out of the Phillips relation. Later, there were many more discoveries of this type, and one could start to think of a SN 1991bg-class. Li et al. (2011b) quantify this class as $15 \%$ of all SNe Ia.

Pakmor et al. (2011) suggest that violent mergers of WDs with a primary of 0.9 
$\mathrm{M}_{\odot}$ reproduce very well the $1991 \mathrm{bg}-$ like SN. Indeed, the simulated optical light curves fit well the data (Hillebrandt et al. 2013). Very recently, the presence of [O I] $\lambda \lambda 6300,6364$ emission in the nebular spectrum of SN 2010lp, suggesting that oxygen is distributed in a non-spherical region close to the centre of the SN ejecta, has also been interpreted as the result of a violent merger (Taubenberger et al. 2013b).

\subsection{2. $C a-$ rich transients}

These events do exhibit unusually strong Ca features at nebular phases, while they look as spectroscopically normal SNe Ia at maximum. Their distribution within their host galaxies shows great similarity with that of the SNe Ia and indicate old progenitor systems (Lyman et al. 2013). According to these authors, they are consistent with helium-shell detonations on low-mass $\mathrm{C}+\mathrm{O}$ white dwarfs. The objects display low peak luminosities, fast photometric evolution, high ejecta velocities, strong Ca emission lines, and they are located in the extreme outskirts of their host galaxies (Kasliwal et al. 2012; see Figure 6).

\subsubsection{Type Iax supernovae}

Foley et al. (2013) identify a new subclass of supernovae called Type Iax. They have low maximum velocities $\left(2000 \lesssim v \lesssim 8000 \mathrm{~km}^{-1}\right.$ ), and typically low peak magnitudes $\left(-14.2 \gtrsim M_{V, p e a k} \gtrsim-18.9 \mathrm{mag}\right)$. In fact, this is the same family of SNe Ia identified by Li et al. (2003) and dubbed SN 2002cx-like SNe Ia. Foley et al. (2013) find that this subclass comprises $31_{-13}^{+17 \%}$. of all SNe Ia. White et al (2014), however, reduce it to $5.6_{-3.7}^{+17}$. Given the large uncertainty, in Figure 9 we have plotted an average of the two estimates.

Those SN2002-cx (or SN Iax) events exhibit iron-rich spectra at early phases, like SN $1991 \mathrm{~T}$ (see Ruiz-Lapuente et al. 1991 for the iron-rich spectra of SN 1991T), a luminosity 


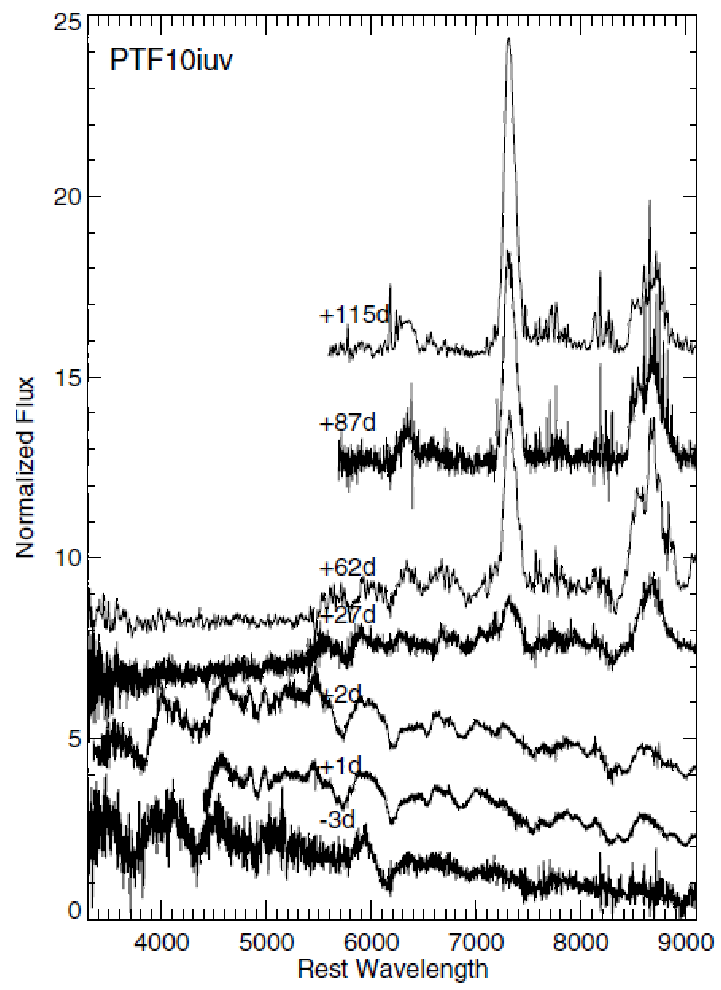

Fig. 6.- Evolution of a Ca-rich transient. It looks like a normal SN Ia at maximum, but it develops a $\mathrm{Ca}$-dominated nebular spectrum. (Courtesy of Mansi Kasliwal; Kasliwal et al. 2012. (CAAS. Reproduced with permission). 


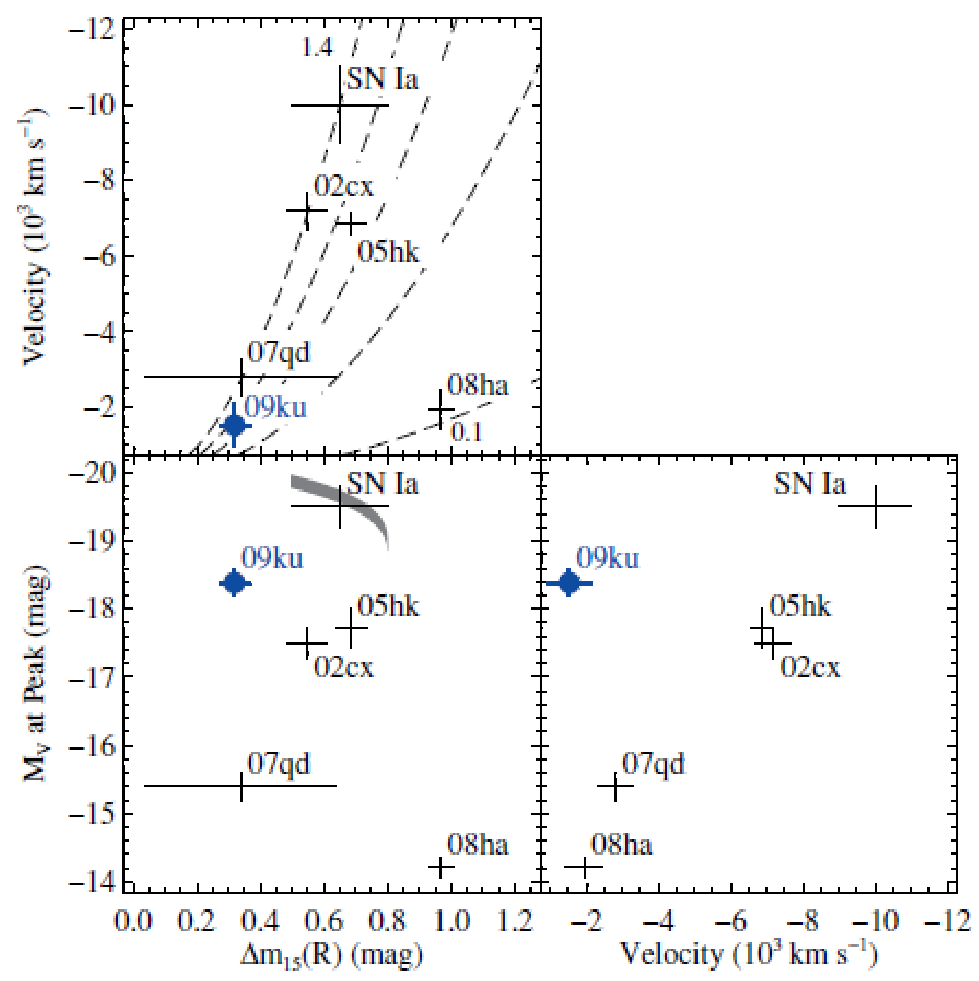

Fig. 7.- Inhomogenety of the properties of SNIax. (Courtesy of Gautham Narayan; Narayan et al. 2011. (c)AAS. Reproduced with permission). 
as low as events like SN 1991bg (i.e. 2 mag below normal Type Ia), and expansion velocities roughly half those of normal SNe Ia. This subclass has a small ${ }^{56} \mathrm{Ni}$ production, as seen at late phases. They move fast into the nebular phase, that giving evidence of the small total mass ejected. A survey of the models able to produce such kind of explosions suggests objects made of a $0.6 \mathrm{M}_{\odot} \mathrm{C}+\mathrm{O} \mathrm{WD}$, with a layer of $\sim 0.17 M_{\odot}$ of He on top, which undergo a He detonation (Li et al. 2003; Wang, Justham \& Han 2013). The origin of the subclass is still under debate, however, because it is quite inhomogeneous (Narayan et al. 2011). White et al. (2014) divide it in two subclasses: the "SN 2002-cx-like" and the "SN 2002es-like" SNe Ia. The former tend to appear in later-type or more irregular hosts, have more varied and generically dimmer luminosities, longer rise times, and they lack a Ti II through in their spectra, when compared to the latter.

The frequency of SN 2002cx events, as compared with normal and SN 1991bg-like events, has also been estimated by Perets et al. (2010). Variants of SN 2002cx events are seen in faint supernovae such as SN 2008ha (Foley et al. 2009, 2010). This last supernova is the faintest member of its subclass. Its late-time photometry is consistent with the production of just a few times $10^{-3} \mathrm{M}_{\odot}$ of ${ }^{56} \mathrm{Ni}$, similar to the estimates from the early light curve (Foley et al. 2009). The small ejecta and ${ }^{56} \mathrm{Ni}$ masses are consistent with a failed deflagration of a WD, that did not disrupt the progenitor (Jordan et al. 2012; Kromer et al. 2013a). There is still another model, proposed earlier, for this particular Type Iax supernova: it is the fallback of a core-collapse supernova (Moriya et al. 2010). This model, then, does not treat SN 2008ha as a thermonuclear supernova, but as the collapse of a $\mathrm{C}+\mathrm{O}$ star of $13 \mathrm{M}_{\odot}$ (model $13 \mathrm{CO} 2$ in their paper). The boundary between the fallback region and the ejecta is determined by whether the velocity of the region exceeds the escape velocity or not. Lyman et al. (2013), argue that the host environments and morphologies point to a generally younger population for this subclass. A model which synthesizes 0.003 $\mathrm{M}_{\odot}$ of ${ }^{56} \mathrm{Ni}$ and ejects $0.074 \mathrm{M}_{\odot}$ of material seems to reproduce the spectra and light curve 
of SN 2008ha. Foley et al. (2013) find the environment of some of these Type Iax SN to be typical of old progenitor systems. They discuss that the most significant reason for their classification of SN 2008ha as a SN Ia is the presence of signatures of the products of thermonuclear processing of $\mathrm{C}+\mathrm{O}$, in particular that of sulfur lines, with an intensity that is only typical of SNe Ia (Foley et al. 2009). Very recently, Foley et al. (2014) report the possible detection of the stellar donor of SN 2008ha in images from the HST. Different possibilities for the progenitor remain open, though the age is constrained to be $<80$ Myr.

Thus, while Type Iax SNe, fall well below the Phillips relation, some of these events should be regarded as subluminous SNe Ia, which might be linked either to He detonations in a shell or to failed deflagrations. Others might originate in core-collapse. The diversity of the events (see Figures 7,8 ) suggests that several mechanisms take place within the sample of SNe Iax, explaining the properties of the different observed events.

\subsection{Rapidly declining SNe Ia}

Perets et al. (2010, 2011a,b; Poznanski et al. 2010) have identified a new subclass of SNe Ia: rapidly declining SNe, such as SN 1885A, 1939C, and 2002bj, that, unlike the Iax subtype, are not faint. SN 2002bj had a short rise time $(<7$ days $)$ and $\Delta m_{15}(B) \sim 3.2$, which is a post-maximum decline much faster than that of the bulk of SNe Ia $\left(\Delta m_{15}(B)<1.7\right)$, and even faster than that of SN $1991 \mathrm{bg}$-like events $\left(1.7<\Delta m_{15}(B)<2\right)$. However, it reached a peak magnitude $M_{B}=-18.5$, which is not faint.

SN 1885A and 1939C arose in old environments, which points to also old WDs as their progenitors. In the case of SN 2002bj, the ejected mass appears to be low $\left(M_{e j}<0.15 M_{\odot}\right)$, that being consistent with the estimated mass of the SN 1885A remnant. Perets et al. (2011b) evaluate the frequency of the SNe in this subclass as being at least 1-2\% of 


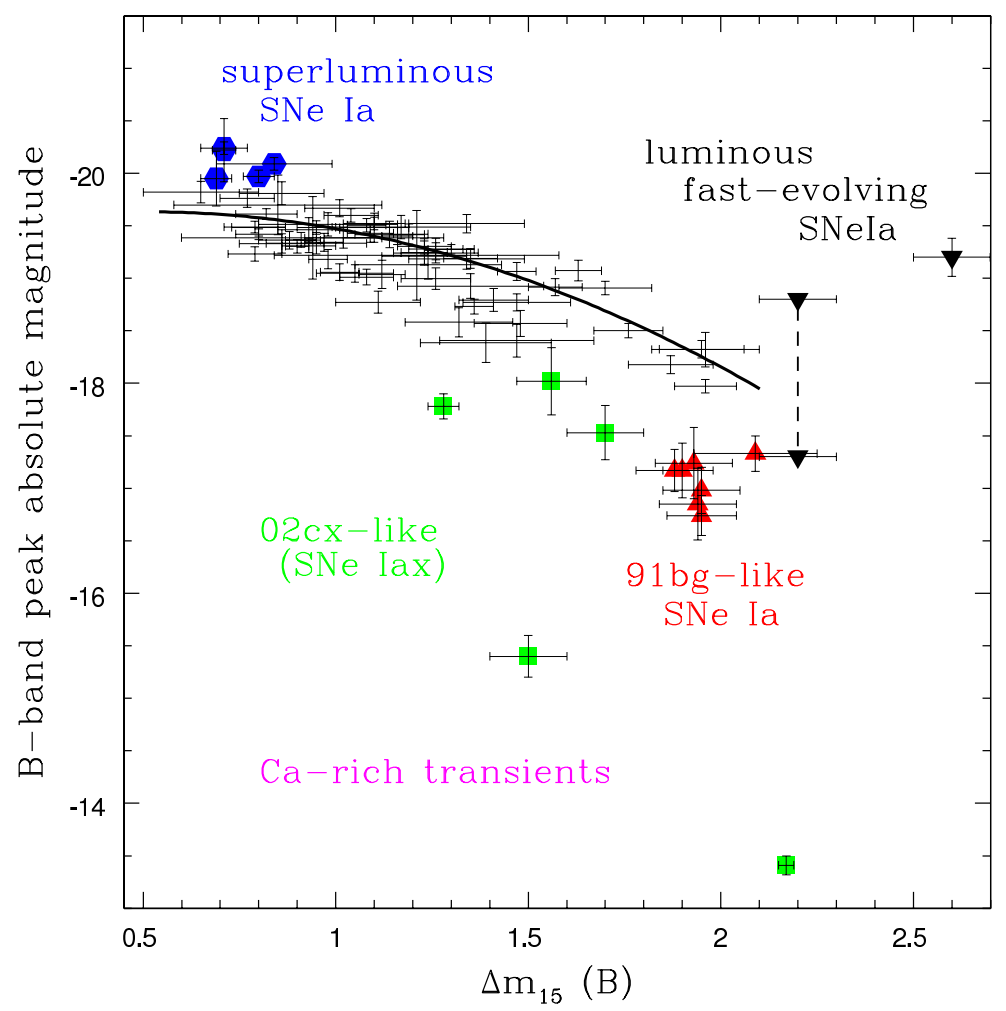

Fig. 8.- The $M_{B}$ vs $\Delta m_{15}(B)$ diagram using the CfA3 sample of 185 SNe Ia (Hicken et al. 2009). The solid line is the Phillips et al. (1999) relation. The fundamental relation developed by Phillips (1993) was modified in Phillips et al. (1999). The figure is adapted from Hillebrandt et al. (2013), and we use the same sources for superluminous and 91bg-like subluminous events. The diversity of Type Iax is taken from Narayan et al. (2011). The area of the Ca-rich transients is marked on the diagram, following Kasliwal et al. (2012). The data on the luminous fast-evolving SNe Ia come from Perets et al. (2011). The proportion in Nature of the different events is tentatively given in Figure 9. 
the global SNe Ia rate. Helium-shell detonations have been suggested as the explosion mechanism (Perets et al. 2010, 2011a; Poznanski et al. 2010), but its consistency with the observations still remains an open question.

\section{The bulk of SNe Ia: cosmological SNe Ia}

It has been presented in section 6 that a significant fraction $(18 \%)$ of the population of SNe Ia show time-variable Na I D features, other features being variable as well. Those supernovae are normal SNe Ia, as the ones that we use for cosmology. Their characteristics point to recurrent novae (Patat 2011) or to systems with significant outflows prior to explosion, as progenitors. Another chunk of the normal (cosmological) SNe Ia likely comes from double-degenerate systems $(\sim 30 \%)$, as suggested by the unburnt $\mathrm{C}$ and $\mathrm{O}$ in the outermost layers (see section 4).

One might ask: how can cosmological SNe Ia have two different origins? The reason is that in both cases the whole $\mathrm{C}+\mathrm{O} \mathrm{WD}$ is burnt, giving rise to the light curve whose variation in peak magnitude is due to the amount of ${ }^{56} \mathrm{Ni}$ synthesized in the explosion, and the line opacity of those events modulates their rate of decline to give $M(B)_{\max }=M(B)_{1.1}+0.786\left[\Delta m_{15}(B)-1.1\right]-0.663\left[\Delta m_{15}(B)-1.1\right]^{2}$, where $M(B)_{1.1}$ is the absolute blue magnitude, at maximum, of a SN Ia with $\Delta m_{15}=1.1$ mag (Phillips et al. 1999). This relation extends the possibility of using SNe Ia with $\Delta m_{15}$ high, since they are seen to make a continuum with the slower ones. Scalzo et al. (2014) derive, from the bolometric light curves of a sample of SNe Ia obtained by the SNFactory, a range of both ejected masses and ${ }^{56} \mathrm{Ni}$ masses. Ejected masses would range, for normal SNe Ia, from 0.9 $\mathrm{M}_{\odot}$ for the fastest decliners to $1.4 \mathrm{M}_{\odot}$ for the slowest ones. The $91 \mathrm{~T}$-like SNe Ia eject

masses in excess of $1.4 \mathrm{M}_{\odot}$ and produce ${ }^{56} \mathrm{Ni}$ masses around $0.8-1.0 \mathrm{M}_{\odot}$. The middle part of the sample is occupied by SNe Ia with Chandrasekhar-mass ejecta and producing 0.6 


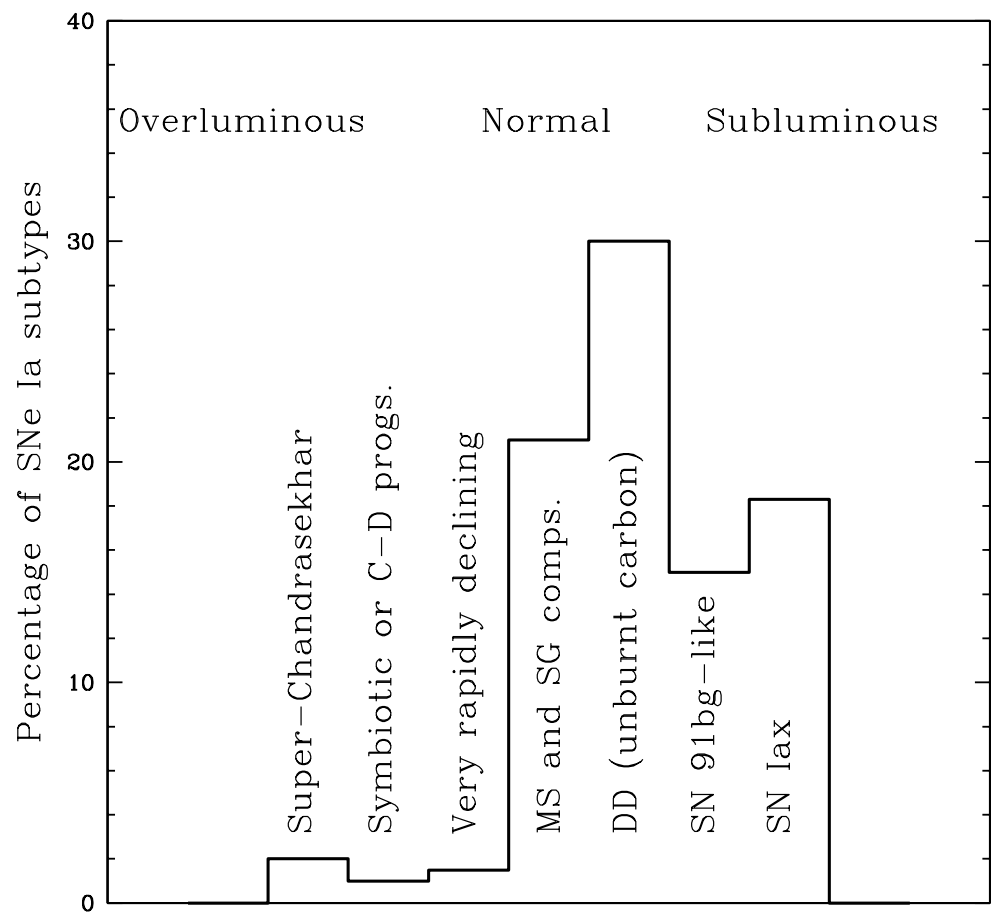

Fig. 9.- Histogram showing the approximate percentages of SNe Ia subtypes. The overluminous super-Chandrasekhar SNe Ia may come from non-violent DD mergings. The small numbers of SN attributed either to symbiotic systems or to the core-degenerate scenario, those showing unburnt $\mathrm{C}$ in their spectra (likely coming from DD mergers), plus those most likely having main sequence stars as mass donors, together with the very fast evolving SNe Ia, have standard luminosities. The SN 1991bg and the SN Iax subtypes are underluminous. The large error bars concerning the fraction of SN of the SN Iax subtype (not shown in the Figure; see text) imply, of course, additional uncertainties on the percentages of the other subtypes, not affecting the relative distribution of percentages amongst them, though. Ca-rich transients are not included, because they are too faint to be considered SNe Ia 
$\mathrm{M}_{\odot}$ of ${ }^{56} \mathrm{Ni}$ (as derived from the nebular spectra of SNe Ia in R96).

It has been argued (Domínguez et al. 2001) that evolutionary effects in the maximumbrightness-rate of decline relation could make difficult the use of SN Ia at very high $z$. Branch et al. (2001) propose an strategy to test evolutionary effects, in the prospect of a space mission devoted to the study of dark energy by means of high-z SNe Ia. Their proposed plan, now completed for large samples of nearby SNe Ia, is to study all possible evolutionary effects in SNe Ia samples at low z. Such effects have now been evaluated from big samples, and it has been shown that they do not to interfere with our understanding of dark energy (Ruiz-Lapuente 2007). The SNe Ia at $z=1.914$ (Jones et al. 2013), at $z=1.71$ (Rubin et al. 2013), and at $z=1.55$ (Rodney et al. 2012) look normal and show no evolutionary effects. Concerning cosmology, it has been seen that these highest-z observations do suppport the $\Lambda C D M$ model of our Universe (see also Conley et al. 2011; Sullivan et al. 2011).

We have learned a lot about normal (cosmological) SNe Ia from the two recent nearby SNe Ia: SN 2011fe and SN 2014J. For the first one, the HST deep images have allowed to discard many single-degenerate scenarios (Li et al. 2011a,b). SN 2011fe showed unburnt $\mathrm{C}$ and $\mathrm{O}$ in the very early spectra. One possible explanation is that it came from a double-degenerate progenitor (see Nomoto, Kamiya \& Nakasato 2013 for an alternative explanation of the unburnt $\mathrm{C}$ and O material). For SN 2014J, it has been possible to rule out red giants as the companions of the $\mathrm{C}+\mathrm{O}$ WD that exploded.

It continues to be safe to exclude SN 1991bg-events, SNe Iax, SNe Ia-CSM and Super-Chandrasekhar SNe Ia (Ca-rich transients as well) from the cosmological samples, as they lay outside the brightness-decline rate relation used in cosmology. They might have been responsible (in particular SNe Iax and SN 1991bg events) for some outliers present in the early samples (such as the first $S C P$ SNe Ia), for which we lacked explanation at those 
epochs of the cosmologically-motivated SNe Ia searches.

Dust absorption in the host galaxy is an important source of systematic error in SN cosmology. A way to minimize its effect on distance determinations is to measure the properties of the $\mathrm{SN}$ in the rest frame infrared. It has been demonstrated (Wood-Vasey et al. 2008; Kirshner 2010; Barone-Nugent et al. 2012) that SNe Ia are better standard candles in the infrared than in the optical wavelengths. The infrared sample of nearby SNe Ia is steadily growing, now (Friedman et al. 2014).

\section{Conclusions}

As we have seen, the last decade has brought considerable progress in the still far from closed search for the progenitor systems of the SNe Ia. A new picture has emerged, where single-degenerate progenitors would now make a much narrower channel than it was thought to be in the 90's. The highlight, here, is that the companions to SNe Ia, at the time of explosion, are very unlikely to be red giants or supergiants, as well as massive main-sequence or subgiant stars. This result comes from searches for companion stars in SNe Ia remnants, in our Galaxy and in the LMC; by looking at pre-explosion images of nearby SNe Ia, like SN 2011fe; from X-ray surveys of SNe Ia made with Swift; from radio observations of nearby SNe Ia, and from confrontation of the early light curves of SNe Ia with theoretical predictions.

On the other hand, the much expanded surveys for transients, at different wavelengths, have found from very dim explosions to overluminous outbursts. Multidimensional modeling of different types of thermonuclear explosions has reached a new level of realism, and they are confronted with observations uncovering the different layers of the exploding objects with unprecedented detail. We still lack, however, the identification of a SNe Ia with the 
dismissal of some previously observed object.

Two different channels (SD and DD) leading to SN Ia explosions still appear to be required, although the balance has now shifted towards the double-degenerate channel, where violent mergings or violent collisions of white dwarfs appear as a promising mechanism. The initial conditions leading to such collisions are still unclear, however. For subluminous SNe Ia of the SNe Iax type, or Ca-rich transients, edge-lit He detonations that might not disrupt the underlying $\mathrm{C}+\mathrm{O} \mathrm{WD}$, or failed deflagrations in the outer shells, seem able to account for the observations and for the rates of the explosions.

From all the preceding, we can reach some tentative conclusions about the fraction of supernovae arising from different kinds of progenitor systems and their explosion mechanisms (see Figure 9).

- The supernovae that show unburnt carbon make $30 \%$ of the SNe Ia class. If we add to that the super-Chandrasekhar explosions (2\%), we can infer that around $32 \%$ of thermonuclear supernovae should arise from the merging of two white dwarfs. Tamped detonations in rapidly rotating white dwarfs may be the explosion mechanism, in the super-Chandrasekhar case, although mergings of massive white dwarfs could also account for these events.

- Between $31_{-13}^{+17} \%$ and $5.6_{-3.7}^{+17}$ of all SNe Ia do belong to the Iax subtype, likely arising either from failed deflagrations or from surface detonations of low-mass white dwarfs. The large error bars come from the uncertainty on the selection effects that work against detection of subluminous events. There are indications that this subclass of SNe Ia with low ejection velocities do, in fact, split into two subclasses. Further research in this are is needed.

- There is a $15 \%$ of SNe Ia of the $91 \mathrm{bg}$-type, which are tentatively associated with 
violent mergings of white dwarfs where the primary is of about $0.9 \mathrm{M}_{\odot}$. Detonation of the $\mathrm{C}+\mathrm{O}$ mixture should then occur.

- The fraction of explosions resulting from the accretion of hydrogen from a red giant star seems to be very low, as metioned before. The SNe coming from a symbiotic progenitor should only make $0.1-1 \%$ of all SNe Ia.

- About $1-2 \%$ of events have luminosities falling well in the middle of the bulk of SNe Ia, but they rise fast and do decline very fast after maximum (the fraction migh be larger, due to selection effects). Their origin remains unclear.

- The remaining fraction of SNe Ia, a $21 \%$, could come from systems made of a $\mathrm{C}+\mathrm{O}$ white dwarf plus a main-sequence or subgiant star, and they could appear, before the explosion, as supersoft X-ray sources or/and recurrent novae. In the SN of this and the former group (as well as in those coming from non-violent DD mergings), the explosion should start as a (generally off-centre) deflagration, to become a detonation in reaching lower-density layers.

Given the large sample of peculiar SNe Ia, it is worth obtaining spectral sequences, when using SN for cosmology. Such project would be made possible by dedicated space missions and by ground-based programs (Wood-Vasey 2010).

\section{Acknowledgments}

I would like to thank Gaston Folatelli, Mario Hamuy, Dan Kasen, Mansi Kasliwal, Gautham Narayan, and Josh Simon, for their kind permissions to use their Figures. My thanks also go to Hagai Perets, Lev Yungelson, Zhengwei Liu, Alexander Tutukov, Thomas Tauris, Enrique García-Berro, Joe Lyman, Raffaella Margutti, Todd Thompson, Mickael Rigault, Markus Kromer, Dan Kasen, Boaz Katz, Mario Hamuy, Michael Wood-Vasey, 
and Marat Gilfanov, for valuable comments, suggestions and criticisms to the first draft of this paper. The useful input from an anonymous referee is also acknowledged. This work has been supported by Grant AYA2012-36353, from the Ministerio de Economía y Competitividad of Spain. 


\section{REFERENCES}

Ade, P.A.R., Aghanim, N., Armitage-Caplan, C., et al. 2013, A\&A, in press, arXiv:1303.5076rv2

Aldering, G., Antilogus, P., Bailey, S., et al. 2006, ApJ, 650, 510

Aldering, G. 2011, private communication

Altavilla, G., Stehle., M., Ruiz-Lapuente, P., et al. 2007, A\&A, 475, 585

Aspden, A.J., Bell, J.B., \& Woosley, S.E. 2010, ApJ, 710, 1654

Badenes, C., \& Maoz, D. 2012, ApJ, 749, L11

Badenes, C., Hughes, J.P., Bravo, E., \& Langer, N. 2007, ApJ, 662, 472

Barbary, K., Aldering, G., Amanullah, R., et al. 2012, ApJ, 745, 32

Baron, E., Hauschildt, P., \& Chen, B. 2009, A\&A, 498, 987

Barone-Nugent, R.L., Lidman, C., Wyithe, J.S.B., et al. 2012, MNRAS, 425, 1007

Bedin, L.R., Ruiz-Lapuente, P., González Hernández, J.I., Canal, R., Filippenko, A.V., \& Méndez, J. 2014, MNRAS, 439, 354

Benetti, S., Cappellaro, E., Turatto, M., Taubenberger, S., Harutyunyan, A., \& Valenti, S. 2006, ApJ, 653, L129

Benz, W., Thielemann F.-K., \& Hills J.G. 1989, ApJ, 342, 986

Benz, W., Cameron, A.G.W., Press, W.H., \& Bowers, R.L. 1990, ApJ, 348, 647

Betoule, M., Kessler, R., Guy, J., et al. 2014, arXiv:1401.4064

Bianco, F.B., Howell, D.A., Sullivan, M., et al. 2011, ApJ, 741, 20 
Bloom, J.S., Kasen, D., Shen, K.J., et al. 2012, ApJ, 744, L17

Bonaparte, I., Matteucci, F., Recchi, S., Spitoni, E., Pipino, A., \& Grieco, V. 2013, MNRAS, 435, 2460

Branch, D., Perlmutter, S., Baron, E., \& Nugent, P. 2001, in Contribution to the SNAP Yellow Book (Snowmass 2001), arXiv: astro-ph/010970

Branch, D., Troxel, M.A, Jeffery, D.J., et al. 2007, PASP, 119, 709

Bravo, E., Tornambé, A., Domínguez, I., \& Isern, J. 1996, A\&A, 306, 811

Chandler, C.J., \& Marvil, J. 2014, Atel 5812.

Chiotellis, A., Schure, K.M., \& Vink, J. 2012, A\&A, 537, 139

Chiotellis, A., Kosenko, D., Schure, K.M., \& Vink, J. 2013, in Binary Paths to Type Ia Supernovae Explosions, ed. R. Di Stefano, M. Orio, \& M. Moe (Cambridge Univ. Press, Cambridge, UK), p. 331

Chomiuk, L., Soderberg, A.M., Moe, M., et al. 2012, ApJ, 750, 164

Churazov, E., Sunyaev, R., Isern, J., et al. 2014, arXiv:1405.3332v1

Conley, A., Howell, D.A., Howes, A., et al. 2006, AJ, 132, 1707

Conley, A., Guy, J., Sullivan, M., et al. 2011, ApJS, 192, 1

Di Stefano, R., \& Nelson, L.A. 1996, in Supersoft X-Ray Sources, ed. J. Greiner (Springer-Verlag, Berlin), p. 3

Di Stefano, R., Voss, R, \& Claeys, J.S.W. 2011, ApJ, 738, L1

Di Stefano, R., \& Kilic, M. 2012, ApJ, 759, 56 
Di Stefano, R., Orio, M., \& Moe, M. (eds.) 2013, Binary Paths to Type Ia Supernovae Explosions (Cambridge: Cambridge Univ. Press, Cambridge UK)

Diehl, R., Siegert, T, Hillebrandt, W., et al. 2014, Science, in press, arXiv:1407.3061

Dilday, B., Howell, D.A., Cenko, S.B., et al. 2012, Science, 337, 942

Domínguez, I., Höflich, P., \& Straniero, O. 2001, ApJ, 557, 279

Dong, S., Katz, B., Kushnir, D., \& Prieto, J.L. 2014, arXiv:1401.3347

Elías-Rosa, N., Greggio, L., \& Botticella, M.T. 2014, Atel 5849

Edwards, Z.I., Pagnotta, A., \& Schaefer, B.E. 2012, ApJ, 747, L19

Filippenko, A.V., Richmond, M.W., Branch, D., et al. 1992, AJ, 104, 1543

Filippenko, A.V. 1997a, ARA\&A, 51, 269

Filippenko, A.V. 1997b, in Thermonuclear Supernovae, ed. P. Ruiz-Lapuente, R. Canal, \& J. Isern (Kluwer, Dordrecht), p. 1

Fink, M., Hillebrandt, W., \& Röpke, F. 2007, A\&A, 476, 1133

Fink, M., Kromer, M., Seitenzahl, I., et al. 2014, MNRAS, 438, 1762

Finzi, A., \& Wolf, R.A. 1967, ApJ, 150, 115

Foley, R.J., Chornock, R., Filippenko, A.V., et al. 2009, AJ, 138, 376

Foley, R.J., Brown, P.J., Rest, A., Challis, P.J., Kirshner, R.P., \& Wood-Vasey, W.M. 2010, ApJ, 708, L61

Foley, R.J., Challis, P.J., Chornock, R., et al. 2013, ApJ, 767, 57

Foley, R.J., Mc Cully, C., Jha, S.W. et al. 2014, arXiv:1408.1091v1 
Folatelli, G., Phillips, M.M., Morrell, N., et al. 2012, ApJ, 745, 74

Friedman, A,S, Wood-Vasey, W.M., Marion, G.H., et al. 2014, arXiv:1408.0465v1

Ganeshalingam, M., Li, W., Filippenko, A.V., et al. 2010, ApJS, 190, 418

Ganeshalingam, M., Li, W., Filippenko, A.V., et al. 2012, ApJ, 751, 142.

García-Senz, D., Cabezón, R.M., Arcones, A., Relaño, A., \& Thielemann, F.-K. 2013, MNRAS, 436, 3413

Geier, S., Marsh, T.R, Wang, B., Dunlap, B., et al. 2013, A\&A, 554, 54

Gilfanov, M., \& Bogdán, A. 2010, Nature, 463, 92

Goldhaber, G., Groom, D.E., Kim, A., et al. 2001, ApJ, 558, 359

González Hernández, J.I., Ruiz-Lapuente, P., Filippenko, A.V., Foley, R.J., Gal-Yam, A., \& Simon, J.D. 2009, ApJ, 691, 1

González Hernández, J.I., Ruiz-Lapuente, P., Tabernero, H.M., Montes, D., Canal, R., Méndez, J., \& Bedin, L.R. 2012, Nature, 489, 533

Goobar, A., Johansson, J., Amanullah, R., et al. 2014, arXiv:1402.0849

Greggio, L. 2005, A\&A, 441, 1055

Guerrero, J., García-Berro, E., \& Isern, J. 2004, A\&A, 413, 257

Hachisu, I. 1986, ApJS, 61, 479

Hachisu, I., Kato, M., \& Nomoto, K. 1996, ApJ, 470, L97

Hachisu, I., Kato, M., Nomoto, K., \& Umeda, H. 1999, ApJ, 519, 314

Hachisu, I., Kato, M., \& Nomoto, K. 2008, ApJ, 683, L127 
Hachisu, I., Kato, M., \& Nomoto, K. 2010, ApJ, 724, L212

Hamuy, M., Phillips, M.M., Suntzeff, N.B., et al. 2003, Nature, 424, 651

Han, Z., \& Podsiadlowski, P. 2004, MNRAS, 350, 1301

Hancock, P.J., Gaensler, B.M., \& Murphy, T. 2011, ApJ, 735, L35

Hayden, B.T., Garnavich, P.M., Kasen, D., et al. 2010, ApJ, 722, 1691

Hicken, M., Garnavich, P.M., Prieto, J.L., Blondin, S., DePoy, D.L., Kirshner, R.P., \& Parrent, J. 2007, ApJ, 669, L17

Hicken, M., Challis, P., Jha, S., et al. 2009, ApJ, 700, 331

Hillebrandt, W., \& Niemeyer, J.C. 2000, ARA\&A, 38, 191

Hillebrandt, W., Kromer, M., Röpke, F.K., \& Ruiter, A.J. 2013, Fr. Phys., 8, 116

Höflich, P. 1991, ApJ, 246, 481

Höflich, P., \& Khokhlov, A.P. 1996, ApJ, 457, 500

Horesh, A., Kulkarni, S.R., Fox, D.B., et al. 2012, ApJ, 746, 21

Hoyle, F., \& Fowler, W.A. 1960, ApJ, 132, 565

Howell, D.A., Sullivan, M, Nugent, P.E. et al. 2006, Nature, 443, 308

Hughes, J.P., Chugai, N., Chevalier, R., Lundqvist, P., \& Schlegel, E. 2007, ApJ. 670, 1260

Iben, I.,Jr., \& Tutukov, A.V. 1984, ApJS, 54, 335

Isern, J., et al. 2013, A\&A, 552, A97

Jeffery, D.J. 1989, ApJS, 71, 951 
Jeffery, D.J. 1990, ApJ, 352, 267

Jones, D., Rodney, S.A., Riess, A.G., et al. 2013, ApJ, 768, 166

Jordan, G.C.IV, Perets, H.G., Fisher, R.T., \& van Rossum, D.R. 2012, ApJ, 761, L23

Kahabka, P., \& van den Heuvel, E.P.J. 1997, ARA\&A, 35, 69

Kasen, D. 2010, ApJ, 708, 1025

Kasliwal, M.M., Kulkarni, S.R., Gal-Yam, A., et al. 2010, ApJ, 723, L98

Kasliwal, M.M., Kulkarni, S.R., Gal-Yam, A., et al. 2012, ApJ, 755, 161

Kato, M., \& Hachisu, I. 1994, ApJ, 437, 802

Katz, B., \& Dong, S. 2012, arXiv:1211.4584

Kerzek, A., Hillebrandt, W., \& Truran, J.W. 1999, A\&A, 345, 831

Kerzendorf, W.E., Schmidt, B.P., Asplund, M., et al. 2009, ApJ, 701, 1665

Kerzendorf, W.E., Schmidt, B.P., Laird, J.B., Podsiadlowski, P., \& Bessell, M.S. 2012, ApJ, 159,7

Kerzendorf, W.E., Yong, D., Schmidt, B.P., et al. 2013, ApJ, 774, 99

Kerzendorf, W.E., Childress, M., Schwächter, J., Do, J., \& Schmidt, B.P. 2014, ApJ, 782, 27

Kirshner, R.P. 2010, in Dark Energy: Observational and Theoretical Approaches, ed. P. Ruiz-Lapuente (Cambridge Univ. Press, Cambridge UK), 151

Kromer, M., Fink, M., Stanishev, V., et al. 2013a, ApJ, 778, L18

Kromer, M., Fink, M., Stanishev, V., et al. 2013b, MNRAS, 429, 2287 
Kushnir, D., Katz, B., Dong, S., Livne, E., \& Fernández, R. 2013, arXiv:1303.1180

Leibundgut, B., Kirshner, R.P., Phillips, M.M., et al. 1993, AJ, 105, 301

Leibundgut, B. 2001, ARA\&A, 39, 67

Leonard, D.C. 2007, ApJ, 670, 1275

Levanon, N., Soker, N., \& Garcia-Berro, E. 2014, arXiv:1408.1375v1

Li, X.-D., \& van den Heuvel, E.P.J. 1997, A\&A, 322, L9

Li, W., Filippenko, A.V., Chornock, R., et al. 2003, PASP, 115, 453

Li, W., Bloom, J.S., Podsiadlowski, P., et al. 2011a, Nature, 480, 348

Li, W., Leaman, J., Chornock, R., et al. 2011b, MNRAS, 412, 1441

Liu, Z.-W., Pakmor, R., Röpke, F.K., et al. 2012, A\&A, 548, 2

Liu, Z.-W., Pakmor, R., Röpke, F.K., et al. 2013, A\&A, 554, A109

Livio, M. \& Truran, J.W. 1992, ApJ, 389, 695

Livio, M., \& Riess, A.G. 2003, ApJ, 594, L93

Livne, E. 1990, ApJ, 354, L53

Livne, E., \& Glasner, A.S. 1991, ApJ, 370, 272

Livne, E., \& Arnett, D. 1995, ApJ, 452, 62

Lü, G., Yungelson, L., \& Han, Z. 2006, MNRAS, 372, 1389

Lü, G., Zhu, C., Wang, Z., \& Wang, N. 2009, MNRAS, 396, 1086 
Lyman, J.D., James, P.A., Perets, H.B., Anderson, J.P., Gal-Yam, A., Mazzali, P., \& Percival, S.M. 2013, MNRAS, 434, 527

Maeda, K., Benetti, S., Stritzinger, M., et al. 2010a, Nature, 466, 82

Maeda, K., Taubenberger, S., Sollerman, J., Mazzali, P.A., Leloudas, G., Nomoto, K., \& Motohara, K. 2010b, ApJ, 708, 1703

Mannucci, F., Della Valle, M., Panagia, N., et al. 2005, A\&A, 433, 807

Maoz, D. \& Mannucci, F. 2012, PASP, 29, 447

Maoz, D., Mannucci, F., \& Nelemans, G. 2014, ARA\&A, in press, arXiv:1312.0628

Margutti, R., Soderberg, A.M., Chomiuk, L., et al. 2012, ApJ, 751, 134

Margutti, R., Parrent, J., Kamble, A., et al. 2014, arXiv:1405.1488

Marietta, E., Burrows, A., \& Fryxell, B. 2000, ApJS, 128, 615

Mazzali, P.A., Chugai, N., Turatto, M., et al. 1997, MNRAS, 284, 151

Mazzali, P.A., Sullivan, M., Hachinger, S., et al. 2014, MNRAS, 439, 1959

McClelland, C.M., Garnavich, P.M., Galbany, L., et al. 2010, ApJ, 720, 704

Meng, X., and Podsiadlowski, P. 2013, ApJ, 778, L35

Mennekens, N., Vanbeveren, D., De Greve, J.P., \& De Donder, E. 2010, A\&A, 515, A89

Mochkovich, R., Guerrero, J., \& Segretain, L. 1997, in Thermonuclear Supernovae, ed. P. Ruiz-Lapuente, R. Canal, \& J. Isern (Kluwer, Dordrecht), p. 187

Moll, R., \& Woosley, S.E. 2013, ApJ, 774, 137

Moll, R., Raskin, C., Kasen, D., \& Woosley, S.E. 2013, arXiv:1311.5008 
Moriya, T., Tominaga, N., Tanaka, M., et al. 2010, ApJ, 719, 1445

Napiwotzki, R., Karl, C.A., Nelemans, G., et al. 2007, in $15^{\text {th }}$ European Workshop on White Dwarfs, ASP Conference Series, 372, ed. R. Napiwotzki \& M.R. Burleigh (Astron. Soc. Pacific, San Francisco), p. 387

Narayan, G., Foley, R.J., Berger, E., et al. 2011, ApJ, 731, L11

Nielsen, M.T.B., Gilfanov, M., Bogdan, A., et al. 2014, arXiv:1402.2896

Nomoto, K. 1982a, ApJ, 253, 798

Nomoto, K. 1982b, ApJ, 257, 780

Nomoto, K., \& Iben, I.,Jr. 1985, ApJ, 279, 531

Nomoto, K., Uenishi, T., Kobayashi, C., Umeda, H., Ohkubo, T., Hachisu, I., \& Kato, M. 2002, in From Twilight to Highlight: the Physics of Supernovae, ed. W. Hillebrandt \& B. Leibundgut (Springer, Berlin), p. 115

Nomoto, K., Kamiya, Y., \& Nakasato, N. 2013, in Binary Paths to Type Ia Supernovae Explosions, ed. R. Di Stefano, M. Orio, \& M. Moe (Cambridge Univ. Press, Cambridge UK), p. 253

Nugent, P.E., Sullivan M., Cenko, S.B., et al. 2011, Nature, 418, 344

Oemler, A.,Jr., \& Tinsley, B.M. 1979, AJ, 84, 985

Orio, M. 2006, ApJ, 643, 844

Pakmor, R., Röpke, F.K., Weiss A., \& Hillebrandt W., 2008, A\&A, 489, 943

Pakmor, R., Kromer, M, Röpke, F.K., Sim, S.A., Ruiter, A.J., \& Hillebrandt, W. 2010, Nature, 463, 61 
Pakmor, R., Hachinger, S., Röpke, F.K., \& and Hillebrandt, W. 2011, A\&A, 528, 117.

Pakmor, R., Kromer, M., Taubenberger, S., Sim, S.A., Röpke, F.K., \& Hillebrandt, W. 2012, ApJ, 747, L10

Pan, K.-C., Ricker, P.M., \& Taam, R.E 2012a, ApJ, 750, 151

Pan, K.-C., Ricker, P.M., \& Taam, R.E 2012b, ApJ, 760, 21

Panagia, N., Van Dyk, S.D., Weiler, K.W., Sramek, R.A., Stockdale, C.J., \& Murata, K.P. 2006, ApJ, 646, 369

Parrent, J.T., Thomas, R.C., Fesen, R.A., et al. 2011, ApJ, 732, 30

Parrent, J.T., Howell, D.A., \& Friesen, B. 2012, ApJ, 752, L26

Patat, F., Benetti, S., Justham, S., et al. 2007, A\&A, 474, 931

Patat, F. 2011, in Binary Paths to Type Ia Supernovae Explosions, ed. R. Di Stefano, M. Orio, \& M. Moe (Cambridge Univ. Press, Cambridge UK), p. 291

Patat, F., Cordiner, M.A., Cox, N.L.J., et al. 2013, A\&A, 54, 162

Perets, H.B., Gal-Yam, A., Mazzali, P.A., et al. 2010, Nature, 465, 322

Perets, H.B., Gal-Yam, A., Crockett, R.M., Anderson, J.P., James, P.A., Sullivan, M., Neill, J.D., \& Leonard, D.C. 2011a, ApJ, 728, L36

Perets, H.B., Badenes, C., Arcevi, I., Simon, J.D., \& Gal-Yam, A. 2011b, ApJ, 730, 89

Perets, H.B., et al. 2012, ApJ, 761, L23

Perlmutter, S., Gabi, S., Goldhaber, G., et al. 1997, ApJ, 483, 565

Perlmutter, S., Aldering, G., Goldhaber, G., et al. 1999, ApJ, 517, 565 
Phillips, M. 1993, ApJ, 413, L105

Phillips, M., Lira, P., Suntzeff, N.B., Schommer, R.A, Hamuy, M., \& Maza, J. 1999, AJ, 118,1766

Piersanti, L., Gagliardi, S., Iben, I.,Jr., \& Tornambé, A. 2003a, ApJ, 583, 885

Piersanti, L., Gagliardi, S., Iben, I.,Jr., \& Tornambé, A. 2003b, ApJ, 598, 1229

Pinto, P.A., \& Eastman, R. G. 2000, ApJ, 530, 744

Podsiadlowski, P. 2003, arXiv:astro-ph/0303660

Poznanski, D., Chornock, R., Nugent, P.E., et al. 2010, Science, 327, 58

Pritchet, C., Howell, D.A., \& Sullivan, M. 2008, ApJ, 683, L25

Raskin, C., Scannapieco, E., Rockefeller, G., Fryer, C., Diehl, S., \& Timmes, F.X. 2010, ApJ, 724, 111

Riess, A.G., Filippenko, A.V., Challis, P., et al. 1998, AJ, 116, 1009

Riess, A.G., Macri, L., Casertano, S., et al. 2011, ApJ, 730, 119

Riess, A.G. 2014, https://webcast.stsci.edu/webcast/

Rigault, M., Copin, Y., Aldering, G., et al. 2013, A\&A, 560, A66

Rodney, S.A., Riess, A.G., et al. 2012, ApJ, 746, 5

Röpke, F.K., Kromer, M., Seitenzahl, I.R., et al. 2012, ApJ, 750, L19

Rubin, D., Knop, R.A., Rykoff, E., et al. 2013, ApJ, 763, 49

Ruiter, A.J., Belczynski, K., \& Fryer, C.L. 2009, ApJ, 699, 2026 
Ruiter, A.J., Belczynski, K., Sim, S.A., Hillebrandt, W., Fryer, C.L., Fink, M., \& Kromer, M. 2011, MNRAS, 417, 408

Ruiter, A.J., Sim, S.A., Pakmor, R., et al. 2013, MNRAS, 429, 1425

Ruiz-Lapuente, P., Cappellaro, E., Turatto, M., et al. 1991, ApJ, 387, L33

Ruiz-Lapuente, P., Jeffery, D., Challis, P.M., et al. 1993, Nature, 365, 728

Ruiz-Lapuente, P. 1996, ApJ, 465, L83

Ruiz-Lapuente, P. 1997, Science, 276, 1813

Ruiz-Lapuente, P., \& Canal, R. 1998, ApJ, 497, L57

Ruiz-Lapuente, P., Comerón, F., Méndez, J., et al. 2004, Nature, 431, 1069

Ruiz-Lapuente, P. 2007, Class. Quant. Grav., 24, R91

Ruiz-Lapuente, P., González Hernández, J.I., Tabernero, H.M., et al. 2014, ESO 093D-0384

Russell, B.R., \& Immler, S. 2012, ApJ, 748, L29

Sahman, D.I., Dhillon, S.D.L., Marsh, T.R., Moll, S., Thoroughgood, T.D., Watson, C.A., \& and Littlefair, S.P. 2013, MNRAS, 433, 1588

Saio, H., \& Nomoto, K. 1985, A\&A, 150, L21

Scalzo, R., Aldering, G., Antilogus, P., et al. 2012, ApJ, 757, 12

Scalzo, R., Aldering, G., Antilogus, P., et al. 2014, arXiv:1402.6842

Schaefer, B.E. 2010, ApJS, 187, 275

Schaefer, B.E., \& Pagnotta, A. 2012, Nature, 481, 164 
Schmidt, W., Ciaraldi-Schoolmann, F., Niemeyer, J.C., Röpke, F.K., \& Hillebrandt, W. 2010, ApJ, 710, 1683

Shappee, B., Kochanek, C.C., \& Stanek, K.Z. 2013, ApJ, 765, 150

Silverman, J.M., Nugent, P.E., Gal-Yam, A., et al. 2013, ApJS, 207, 3

Sim, S., Röpke, F.K., Kromer, M., et al. 2013, in Binary Paths to Type Ia Supernovae Explosions, ed. R. Di Stefano, M. Orio, \& M. Moe (Cambridge Univ. Press, Cambridge, UK), p. 267

Simon, J.D., Gal-Yam, A., Gnat, O., et al. 2009, ApJ, 702, 1157

Sternberg, A., Gal-Yam, A., Simon, D.C., et al. 2011, Science, 333, 856

Soker, N. 2013, in Binary Paths to Type Ia Supernovae Explosions, ed. R. Di Stefano, M. Orio, \& M. Moe (Cambridge Univ. Press, Cambridge, UK), p. 72

Soker, N., Kashi, A., García-Berro, E., Torres, S., \& Camacho, J. 2013, MNRAS, 431, 1541

Soker, N., García-Berro, E., \& Althaus, L.G. 2014, arXiv:1309.0368v2

Soraisam, M.D., \& Gilfanov, M. 2014, arXiv:1401.6148v1

Sparks, W.M., \& Stecher, T.P. 1974, ApJ, 188, 149

Starrfield, S., Sparks, W.M, \& Shaviv, G. 1988, ApJ, 325, L35

Sullivan, M., Guy, J., Conley, A., et al. 2011, ApJ, 737, 102

Taam, R.E. 1980, ApJ, 237, 142

Taubenberger, S., Benetti, S., Childress, M., et al. 2011, MNRAS, 412, 2735

Taubenberger, S., Kromer, S., Pakmor, R., et al. 2013a, ApJ, 775, L43 
Taubenberger, S., Kromer, M., Hachinger, S., et al. 2013b, MNRAS, 432, 3117

The, L-S., \& Burrows, A. 2014, ApJ, 786, 141.

Thomas, R.C., Aldering G., Antilogus P., et al. 2011, ApJ, 743, 27

Thompson, T. 2011, ApJ, 741, 82

Tornambé, A., \& Piersanti, L. 2013, MNRAS, 431, 1812

Totani, T., Morokuma, T., Oda, T., Doi, M., \& Yasuda, N. 2008, PASJ, 60, 1327

Tutukov, A.V., \& Yungelson, L.R. 1979, Acta Astron., 29, 665

van den Heuvel, E.P.J., Bhattacharya, D., Nomoto, K., \& Rappaport, S.A. 1992, A\&A, 262,97

Wang, B., Meng, X., Chen, X., \& Han, Z. 2009, MNRAS 395, 847

Wang, B., \& Han, Z. 2012, New Astron. Revs., 56, 122

Wang, B., Justham, S., \& Han, Z. 2013, arXiv:1310.2297v1

Wang, L., \& Wheeler, J.C. 2008, ARA\&A, 46, 433

Webbink, R.F. 1984, ApJ, 277, 355

Wheeler, J.C., \& Hansen, C.J. 1971, Ap\&SS, 11, 373

Whelan, J., \& Iben, I.,Jr. 1973, ApJ, 186, 1007

White, C.J., Kasliwal, M.M., Nugent, P.E., et al. 2014, arXiv:1405.7409v1

Wood-Vasey, W. M., Friedman, A.S., Bloom, J.S., et al. 2008, ApJ, 689, 377

Wood-Vasey, W.M. 2010, in Dark Energy: Observational and Theoretical Approaches, ed. P. Ruiz-Lapuente (Cambridge Univ. Press, Cambridge UK), 202 
Woosley, S.E., Taam, R.E., \& Weaver, T.A. 1986, ApJ, 301, 601

Woosley, S.E., \& Weaver, T.A. 1994, ApJ, 423, 371

Woosley, S.E. 2007, ApJ, 668, 1109

Woosley, S.E., Kerstein, A.R., \& Aspden, A.J. 2011, ApJ, 734, 37

Woosley, S.E., \& Kasen, D. 2011, ApJ, 734, 38

Yoon, S.-C., \& Langer, N. 2005, A\&A, 435, 967

Yungelson, L., Livio, M., Truran, J.W., Tutukov, A., \& Fedorova, A. 1996, ApJ, 466, 890

Zheng, W., Shivvers, I., Filippenko, A.V., et al. 2014, arXiv:1401.7968

This manuscript was prepared with the AAS LATEX macros v4.0. 